\title{
Spatial Modulated Multicarrier Sparse Code-Division Multiple Access
}

\author{
Yusha Liu, Student Member, IEEE, Lie-Liang Yang, FIEEE, Pei Xiao, SM IEEE, Harald Haas, FIEEE and Lajos \\ Hanzo, FIEEE
}

\begin{abstract}
This paper proposes a novel spatial-modulated multicarrier sparse code-division multiple access (SM/MC-SCDMA) system for achieving massive connectivity in device-centric wireless communications. In our SM/MC-SCDMA system, the advantages of both MC signalling and SM are amalgamated to conceive a low-complexity transceiver. Sparse frequency-domain spreading is utilized to mitigate the peak-to-average power ratio (PAPR) of MC signalling, as well as to facilitate low-complexity detection using the message passing algorithm. We then analyze the single-user bit error rate performance of SM/MC-SCDMA systems communicating over frequency-selective fading channels. Furthermore, the performance of SM/MC-SCDMA systems is evaluated based on both Monte-Carlo simulations and analytical results. We demonstrate that our low-complexity SM/MCSCDMA transceivers are capable of achieving near-maximum likelihood (ML) performance even when the normalized userload is as high as two, hence constituting a variable solution to support massive connectivity in device-centric wireless systems.
\end{abstract}

Index Terms-Multicarrier, CDMA, sparse spreading, spatial modulation, maximum a posteriori detection, message passing algorithm, performance.

\section{INTRODUCTION}

In recent years, sparse code division multiple-access (SCDMA), one of the most popular non-orthogonal multipleaccess (NOMA) schemes, has been considered as a promising next-generation (5G) candidate technique [1-7]. In contrast to the classic CDMA, where user data are spread over all chips simultaneously [8], in a SCDMA system, each user's data is spread using sparse time-domain or frequency-domain spreading sequences [2]. For example, a low density signature (LDS) based technique has been proposed in [1] to exploit the beneficial properties of sparse signatures for approaching the optimum performance in systems supporting a high userload by relying on the massage passing algorithm (MPA) $[9$, 10]. Further studies include the design of sparse code multipleaccess (SCMA) [3], which amalgamates modulation, spreading as well as constellation shaping into a single codebook. Explicitly, the data are no longer carried only by the amplitude

Y. Liu, L.-L. Yang and L. Hanzo are with School of Electronics and Computer Science, University of Southampton, SO17 1BJ, UK. (E-mail: yl6g15, 1ly, 1h@ecs.soton.ac.uk)

Pei Xiao is with the Centre for Communication Systems Research (CCSR), Faculty of Engineering and Physical Sciences, University of Surrey Guildford, GU2 7XH, UK. (E-mail: p.xiao@surrey.ac.uk).

Harald Haas is with the Institute for Digital Communications (IDCOM), University of Edinburgh, Edinburgh, EH9 3JL, U.K. (E-mail: h.haas@ed.ac.uk).

The financial support of the EPSRC projects EP/P034284/1, EP/P03456X/1 and of the ERC's Advance Fellow Award QuantCom are gratefully acknowledged. and phase modulated (APM) symbols but by specifically designed multi-dimensional codewords. Hence, additional coding gain becomes achievable by the proposed design of the code books mapping the data to the codewords. We also note that powerful yet low-complexity receiver designs based on the MPA have been proposed in [11-16], which are capable of reducing the detection complexity at the cost of a modestly degraded BER performance in comparison with the optimum detection based on maximum likelihood detection (MLD).

On the other hand, spatial modulation (SM) [17-24] has been regarded as a promising low-complexity multiple-input multiple-output (MIMO) technique, activating a single antenna or a fraction of all transmit antennas (TAs) at a time. Specifically, in SM, the transmitter encodes part of the information bits into the active TA indices by space-shift keying (SSK) [25, 26], while the activated TAs can further convey some information by conventional APM, such as phase shift keying (PSK) or quadrature amplitude modulation (QAM). In this way, the overall throughput can be increased by the extra SSK modulated bits.

SM has also been studied in conjunction with multipleaccess (MA) communications [27-32]. Specifically, in [27], SM has been combined with spatial division multiple access (SDMA), where both single-user and multiuser interference-aware detectors were considered. In [28], the bit error ratio (BER) performance of SSK-modulated or generalized SSK (GSSK)-modulated SDMA systems employing single-user detection or multiuser MLD has been investigated. In [29], a SSK-modulated antenna-hopping SDMA system and a range of linear as well as non-linear multiuser detectors (MUD) were proposed. By exploiting the sparsity of the SM signals, in [30], compressive sensing (CS) aided detection algorithms have been proposed for large-scale SDMA systems. Zheng [31] has also conceived a massive SM aided MIMO system, and proposed an efficient search algorithm for signal detection. Furthermore, in [32], massive MIMO systems combined with generalized SM (GSM) have been proposed relying on two MPAs for parallel interference cancellation.

Furthermore, in order to support large-scale connectivity in the next generation communication systems, SM has been studied in the context of the NOMA principle [33-37]. In more detail, a joint NOMA-SM transmission scheme has been proposed in [33] for attaining an increased bandwidth efficiency in downlink time-varying channels. In [34], a cooperative vehicle-to-vehicle NOMA combined scheme with SM has been introduced and its performance has been investigated. Furthermore, in [35], the authors have proposed and investi- 
gated a general NOMA downlink scheme relying on SM. By contrast, a SM-assisted two-user NOMA uplink scheme based on SDMA principles has been studied in [36]. Additionally, in [37], a low-complexity SCDMA system combined with SM has been proposed for supporting heavily loaded machine-type communications.

In the above-mentioned references on SM aided uplink MA communications, Siregar et al. [36] have only considered a two-user scenario. The SM-SCDMA scheme studied in [37] is capable of supporting heavily loaded MA by assigning different users sparse codes for direct-sequence (DS) spreading. However, frequency-flat fading has been assumed in [37]. If practical frequency-selective fading channels are considered, SM-SCDMA will suffer from inter-symbol interference (ISI), hence the complexity of the MPA-relied detector (MPAD) is exponentially increased with the number of paths of the frequency-selective fading channels. It is widely recognised that an effective solution to combat frequency-selective fading is to employ multicarrier (MC) signalling [38], which is capable of exploiting the energy scattered across the frequencydomain via combining the signals gleaned from different subcarriers. Furthermore, MC signalling benefits from the employment of efficient fast Fourier transform (FFT) based modulation techniques for low-complexity implementation. However, MC signalling has the disadvantage of high peakto-average power ratio (PAPR), making its employment in the uplink energy-inefficient, which becomes critical in devicecentric communications. To be more specific, for a conventional MC system simultaneously activating all its $N$ subcarriers, the PAPR is $N[38,39]$. By contrast, for a MCSCMA system activating only $d_{f}$ out of its $N$ subcarriers, the PAPR is only $d_{f}$. Here, it is worth noting that $d_{f}$ in SCMAassisted systems is usually a very small value, typically 2 or 3 , regardless of the value of $N[1,37]$. Hence, the SCMA-assisted MC schemes are capable of mitigating the PAPR problem of conventional MC systems.

Bearing in mind the above-mentioned issues, we exploit the joint space-, time-, and frequency-domain resources for transmission over frequency-selective fading channels. Explicitly, we propose a novel SM-aided MC SCDMA (SM/MCSCDMA) system employing LDS spreading sequences, in order to support multiuser communications in ultra-dense deployments requiring massive connectivity. It is shown that our proposed SM/MC-SCDMA scheme is capable of circumventing the aforementioned problems, due to its following merits. Firstly, in contrast to [37], the SM/MC-SCDMA system incorporating $\mathrm{MC}$ signalling exploits the frequency-selective fading to achieve frequency diversity. Secondly, by introducing sparse code based spreading in the frequency-domain, which results in only a very small fraction (typically 2 or 3 ) of subcarriers being activated by each user, the PAPR problem can be efficiently mitigated. This is because for a $N$-subcarrier MC system, the PAPR is $N$ [38]. By contrast, when $d_{x}$ sparse codes are employed, the PAPR is reduced to $d_{x}$, which is usually very small, as above-mentioned. Furthermore, similar to [37], employing sparse spreading allows a SM/MCSCDMA system to achieve near-optimum BER performance, even when it is heavily loaded with a normalized loading factor as high as two. Hence, the proposed SM/MC-SCDMA scheme with MPAD exhibits all the compelling characteristics required for supporting massive connectivity in device-centric communications.

Against this backdrop, the novel contributions of this paper are summarized below.

1) A SM/MC-SCDMA system is proposed for supporting massive uplink connectivity. In the proposed system, SM is employed for reducing the number of radio frequency (RF) chains. In contrast to the SM-SCDMA system proposed in [37], which assumes flat fading, the SM/MCSCDMA employs MC signalling to combat frequencyselective fading. Sparse spreading is employed for the sake of facilitating low-complexity detection, whilst significantly alleviating the PAPR problem of MC systems. Furthermore, a MPAD is developed for the SM/MCSCDMA system for low-complexity detection, even in the face of a high normalized user-load.

2) Since our proposed SM/MC-SCDMA system is designed for operation in practical frequency-selective fading channels, which result in correlated fading in the frequency-domain, the single-user BER bounds derived in [40-43] cannot be directly exploited, since all of them assume independent flat Rayleigh fading. Therefore, we analyze the single-user BER bound of the SM/MCSCDMA system, when assuming that the signals experience frequency-selective fading, whilst taking into account the correlation among the subcarriers.

3) Based on the single-user BER bound, we propose the guidelines for sparse code design. Furthermore, we conceive a sparse code allocation technique for achieving a high diversity gain.

4) The BER performance of the proposed SM/MCSCDMA system using MPAD is studied both by MonteCarlo simulations and by our analytical results. Additionally, the SM/MC-SCDMA scheme is generalized to the SM/MC-SCMA arrangement for the sake of obtaining extra shaping gain. Furthermore, the BER performance of both SM/MC-SCDMA and SM/MCSCMA is compared to that of other related legacy MIMO schemes.

The rest of this paper is structured as follows. Section II describes the transmitter and receiver schematics of the proposed SM/MC-SCDMA system. Different detection algorithms are detailed in Section III, whereas the analysis of the singleuser BER bound of SM/MC-SCDMA system is provided in Section IV. Section V characterizes the BER performance of the SM/MC-SCDMA systems in different scenarios. Finally, our main conclusions are summarized in Section VI.

\section{DESCRIPTION OF THE SM/MC-SCDMA SYSTEM}

In this section, we describe the SM/MC-SCDMA system model, which includes the transmitter model of Section II-A and the receiver of Section II-B. In the SM/MC-SCDMA system considered, we assume that the number of subcarriers $N$ is significantly higher than the number of resolvable time-domain paths $L$, in line with the MC systems' typical design [39]. 
Hence, the individual subcarriers experience the flat fading, but the adjacent subcarriers may experience correlated attenuation. The other assumptions and notations will be detailed along with our discussions.

\section{A. Transmitter Model}

We investigate a single-cell uplink MC communication system, where $K$ users simultaneously transmit their information to a BS over frequency-selective fading channels in the timedomain. We assume that each user employs $M_{1}$ TAs, while the BS employs $U$ receive antennas (RAs). For simplicity, during a symbol period, each user sends a symbol by activating one of the $M_{1}$ TAs to transmit an $M_{2}$-ary APM $\left(M_{2} \mathrm{APM}\right)$ symbol using SM [17]. Our scheme can be readily extended to activating multiple TAs to convey multiple APM symbols $[19,20]$. Therefore, as in $[17,37]$, the symbol conveyed by the indices of the $M_{1}$ TAs is referred to as the SSK symbol, which assumes a value from $\mathcal{S}_{1}=\left\{0,1, \ldots, M_{1}-1\right\}$, and has $b_{1}=\log _{2} M_{1}$ bits per symbol (BPS). Meanwhile, the $b_{2}=\log _{2} M_{2}$ bits of an $M_{2}$-ary APM symbol takes a value from $\mathcal{S}_{2}=\left\{s_{2,0}, s_{2,1}, \ldots, s_{2, M_{2}-1}\right\}$. Furthermore, we assume that the elements in $\mathcal{S}_{2}$ are normalized to satisfy $\sum_{i=0}^{M_{2}-1}\left|s_{2, i}\right|^{2} / M_{2}=1$.

Fig. 1 represents the transmitter schematic of the $k$ th user in a SM/MC-SCDMA system, where the $b$-bit symbol $\boldsymbol{b}_{k}$ of user $k$ is first divided into two sub-symbols, $\boldsymbol{b}_{k 1}$ and $\boldsymbol{b}_{k 2}$. Explicitly, $\boldsymbol{b}_{k 1}$ is mapped by $f_{1}\left(\boldsymbol{b}_{k 1}\right)$ to give a $b_{1}$-bit SSK symbol $s_{k 1} \in \mathcal{S}_{1}$, which activates a TA. By contrast, $f_{2}\left(\boldsymbol{b}_{k 2}\right)$ maps $\boldsymbol{b}_{k 2}$ to an $M_{2}$-ary APM symbol $s_{k 2} \in \mathcal{S}_{2}$, which is first spread by a sparse code of length $N$ assigned to user $k$. Let the spreading code assigned to user $k$ be expressed as $\boldsymbol{c}_{k}=\left[c_{k 0}, c_{k 1}, \ldots, c_{k(N-1)}\right]^{T}$, which is normalized to satisfy $\left\|\boldsymbol{c}_{k}\right\|^{2}=1$ [1]. Then, the spread-spectrum signal $s_{k 2} \boldsymbol{c}_{\boldsymbol{k}}$ is IFFT transformed to the time-domain, which is followed by the parallel-to-serial $(\mathrm{P} / \mathrm{S})$ conversion. Finally, after adding a cyclic prefix (CP) of sufficient length, the SM/MC-SCDMA signal is transmitted from the $s_{k 1}$-th TA activated by the $M_{1}$ SSK symbol $s_{k 1}$.

For the sparse spreading codes, we assume $d_{x}<<N$ to be the maximum number of chips that a user's signal spreads over. Correspondingly, the number of users sharing one of the $N$ chips is denoted by $d_{c}$, which has the property of $d_{c}<<K$. For simplicity, we set both $d_{x}$ and $d_{c}$ to constants, implying that regular spreading codes are employed in our SM/MCSCDMA system.

Additionally, for the convenience of our ensuing discussions, the symbols transmitted by $K$ users is expressed as $\boldsymbol{x}=\left[x_{0}, x_{1}, \cdots, x_{K-1}\right]^{T}$, where $x_{k}$ is $b=b_{1}+b_{2}$ bits contributed by both the $M_{1}$-ary SSK symbol and the $M_{2}$-ary APM symbol. Hence, $x_{k} \in \mathcal{S}=\left\{\mathcal{S}_{1} \otimes \mathcal{S}_{2}\right\}=\left\{s_{0}, s_{1}, \cdots, s_{M-1}\right\}$, which is a set consisting of all the $M=M_{1} M_{2}$ different combinations of the elements in $\mathcal{S}_{1}$ and those in $\mathcal{S}_{2}$.

\section{B. Receiver Model}

Given the channel impulse response (CIR) between the $s_{k 1}$ th TA of user $k$ and the $u$ th RA of the BS as

$$
\begin{aligned}
\boldsymbol{h}_{s_{k 1}}^{(u)}= & {\left[h_{s_{k 1}, 0}^{(u)}, h_{s_{k 1}, 1}^{(u)}, \cdots, h_{s_{k 1}, L-1}^{(u)}\right]^{T}, s_{k 1}=0, \ldots, M_{1}-1 ; } \\
& u=1,2, \cdots, U ; k=0,1, \cdots, K-1,
\end{aligned}
$$

the corresponding frequency-domain channel transfer function (FDCHTF) experienced by the $N$ subcarriers can be expressed as [38]

$$
\hat{\boldsymbol{h}}_{s_{k 1}}^{(u)}=\mathcal{F} \boldsymbol{\Phi}_{L} \boldsymbol{h}_{s_{k 1}}^{(u)}
$$

where $\Phi_{L}$ is a $(N \times L)$ mapping matrix constituted by the first $L$ columns of an identity matrix $\boldsymbol{I}_{N}$, and $\mathcal{F}$ is the $(N \times N)$ FFT matrix having the property of $\mathcal{F F}^{H}=\mathcal{F}^{H} \mathcal{F}=N \boldsymbol{I}_{N}$.

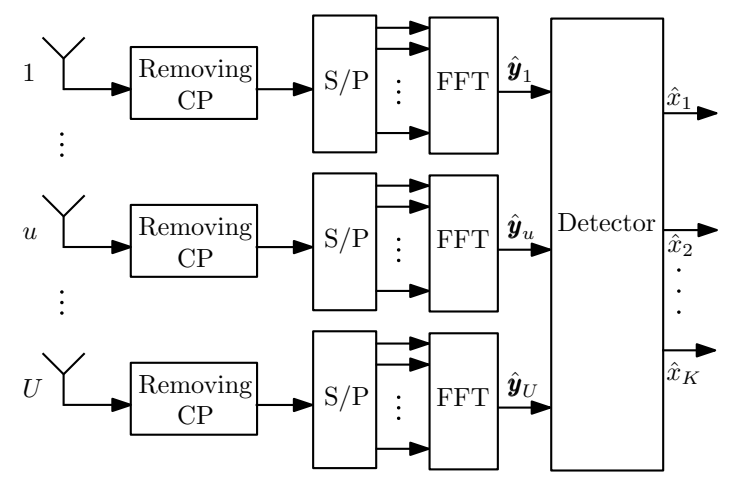

Fig. 2. The receiver schematic diagram of $u$ th antenna and $k$ th user in the SM/MC-SCDMA system.

The receiver schematic of the SM/MC-SCDMA system is shown in Fig. 2, where the CP removal, S/P conversion and the FFT blocks are conventional, which follow [38]. Consequently, after the FFT-based detection operation to transform the timedomain signals to the frequency-domain, the observations obtained from the $N$ subcarriers of the $u$ th RA can be expressed as

$$
\boldsymbol{y}_{u}=\sum_{k=1}^{K} \boldsymbol{C}_{k} \hat{\boldsymbol{h}}_{s_{k 1}}^{(u)} s_{k 2}+\boldsymbol{n}_{u}, \quad u=0,1, \cdots, U-1
$$

where we have $\boldsymbol{C}_{k}=\operatorname{diag}\left\{\boldsymbol{c}_{k}\right\}$, the noise vector $\boldsymbol{n}_{u}$ obeys the zero-mean Gaussian distribution with a covariance matrix of $2 \sigma^{2} \boldsymbol{I}_{N}$, expressed as $\mathcal{C N}\left(0,2 \sigma^{2} \boldsymbol{I}_{N}\right)$, where $\sigma^{2}=1 /(2 \gamma)$. Furthermore, $\gamma=b \gamma_{0}$ denotes the signal-to-noise ratio (SNR) per symbol, while $\gamma_{0}$ is the SNR per bit. Let $\boldsymbol{y}=\left[\boldsymbol{y}_{0}^{T}, \boldsymbol{y}_{1}^{T}, \cdots, \boldsymbol{y}_{U-1}^{T}\right]^{T}$, $\hat{\boldsymbol{h}}_{s_{k 1}}=\left[\left(\hat{\boldsymbol{h}}_{s_{k 1}}^{(0)}\right)^{T},\left(\hat{\boldsymbol{h}}_{s_{k 1}}^{(1)}\right)^{T}, \cdots,\left(\hat{\boldsymbol{h}}_{s_{k 1}}^{(U-1)}\right)^{T}\right]^{T}$ and $\boldsymbol{n}=$ $\left[\boldsymbol{n}_{0}^{T}, \boldsymbol{n}_{1}^{T}, \cdots, \boldsymbol{n}_{U-1}^{T}\right]^{T}$. Note that all these vectors are $U N$ - 


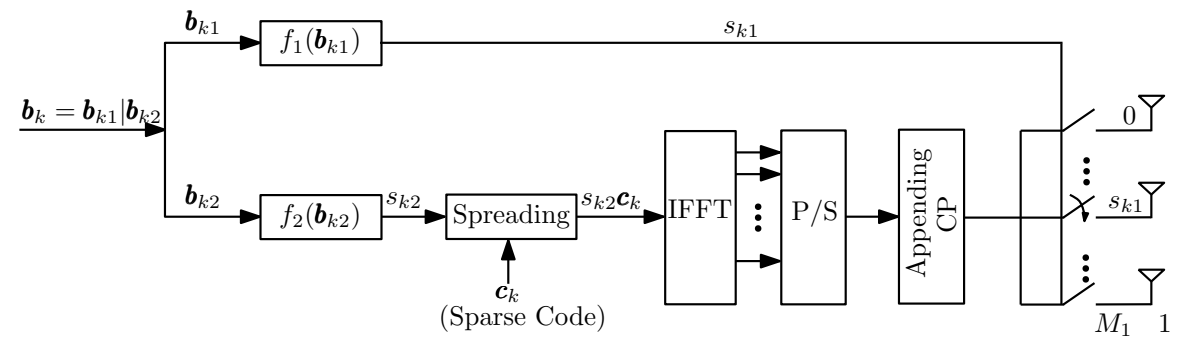

Fig. 1. The transmitter schematic diagram of the $k$ th user in the SM/MC-SCDMA system.

dimensional. Then, it can be shown that we have

$$
\begin{aligned}
\boldsymbol{y} & =\sum_{k=1}^{K}\left(\boldsymbol{I}_{U} \otimes \boldsymbol{C}_{k}\right) \hat{\boldsymbol{h}}_{s_{k 1}} s_{k 2}+\boldsymbol{n} \\
& =\sum_{k=1}^{K}\left(\boldsymbol{I}_{U} \otimes \boldsymbol{C}_{k}\right) \hat{\boldsymbol{H}}_{k} \boldsymbol{e}_{s_{k 1}} s_{k 2}+\boldsymbol{n} \\
& =\sum_{k=1}^{K} \boldsymbol{H}_{k} \boldsymbol{e}_{s_{k 1}} s_{k 2}+\boldsymbol{n} \\
& =\boldsymbol{H} \boldsymbol{e}_{s_{1}} \boldsymbol{s}_{2}+\boldsymbol{n} .
\end{aligned}
$$

In the above equations, $\otimes$ denotes the Kronecker product [44], and we have $\hat{\boldsymbol{H}}_{k}=\left[\hat{\boldsymbol{h}}_{0 k}, \hat{\boldsymbol{h}}_{1 k}\right.$,

$\left.\cdots, \hat{\boldsymbol{h}}_{\left(M_{1}-1\right) k}\right]$, which is $\left(U N \times M_{1}\right)$-dimensional, where $\hat{\boldsymbol{h}}_{m k}$ is in the form of $\hat{\boldsymbol{h}}_{s_{k 1}}$ defined above and with $s_{k 1}=m$, while $\boldsymbol{e}_{s_{k 1}}$ is a $M_{1}$-length vector with the $s_{k 1}$ th element being 1 . The rest of the elements are zeros. Still referring to (4), we have $\boldsymbol{H}_{k}=\left(\boldsymbol{I}_{U} \otimes \boldsymbol{C}_{k}\right) \hat{\boldsymbol{H}}_{k}, \boldsymbol{H}=\left[\boldsymbol{H}_{1}, \boldsymbol{H}_{2}, \cdots, \boldsymbol{H}_{K}\right]$, which is a $\left(U N \times M_{1} K\right)$-dimensional matrix, and finally, we have $\boldsymbol{e}_{s_{1}}=\left[\boldsymbol{e}_{s_{11}}^{T}, \boldsymbol{e}_{s_{21}}^{T}, \cdots, \boldsymbol{e}_{s_{K 1}}^{T}\right]^{T}$ and $\boldsymbol{s}_{2}=\left[s_{12}, s_{22}, \cdots, s_{K 2}\right]^{T}$. We consider the signal detection in the sequel.

\section{Signal Detection In SM/MC-SCDMA Systems}

In this section, we first consider the optimal MLD in Section III-A, followed by the optimal MAPD in Section III-B. Then, a low-complexity near-optimal detector based on message passing algorithm (MPA) is introduced in Section III-C.

\section{A. Maximum-Likelihood Detection (MLD)}

The MLD detects the symbols transmitted by $K$ users based on the maximum likelihood principle. For clarity, in this paper, $x, \tilde{x}$ and $\hat{x}$ represent a transmitted symbol, a symbol hypothesized by the search algorithms and a detected symbol, respectively. Based on (4), the MLD finds the estimate of $x$ by solving the optimization problem of

$$
\begin{aligned}
\hat{\boldsymbol{x}}= & \arg \min _{\tilde{\boldsymbol{x}} \in \mathcal{S}^{K}}\left\{\left\|\boldsymbol{y}-\sum_{k=1}^{K}\left(\boldsymbol{I}_{U} \otimes \boldsymbol{C}_{k}\right) \hat{\boldsymbol{h}}_{\tilde{s}_{k 1}} \tilde{s}_{k 2}\right\|^{2}\right\} \\
= & \arg \max _{\tilde{\boldsymbol{x}} \in \mathcal{S}^{K}}\left\{\sum_{k=1}^{K} \Re\left\{\tilde{s}_{k 2}^{*} \hat{\boldsymbol{h}}_{\tilde{s}_{k 1}}^{H}\left(\boldsymbol{I}_{U} \otimes \boldsymbol{C}_{k}\right)^{H} \boldsymbol{y}\right\}\right. \\
& \left.-\frac{1}{2} \sum_{k=1}^{K} \sum_{i=1}^{K} \tilde{s}_{k 2}^{*} \hat{\boldsymbol{h}}_{\tilde{s}_{k 1}}^{H}\left(\boldsymbol{I}_{U} \otimes \boldsymbol{C}_{k}^{H} \boldsymbol{C}_{i}\right) \hat{\boldsymbol{h}}_{\tilde{s}_{i 1}} \tilde{s}_{i 2}\right\},
\end{aligned}
$$

where $\tilde{x}_{k}=\tilde{s}_{k 1} \mid \tilde{s}_{k 2}$ represents a composite SSK-APM symbol transmitted by user $k$, determining the corresponding CIR $\hat{h}_{\tilde{s}_{k 1}}$ and $\tilde{s}_{k 2}$ used in (5), while $\Re\{a\}$ returns the real part of $a$. It can be readily shown that in (5), we have:

$$
\begin{gathered}
\hat{\boldsymbol{h}}_{\tilde{s}_{k 1}}^{H}\left(\boldsymbol{I}_{U} \otimes \boldsymbol{C}_{k}\right)^{H} \boldsymbol{y}=\sum_{u=0}^{U-1}\left(\hat{\boldsymbol{h}}_{\tilde{s}_{k 1}}^{(u)}\right)^{H} \boldsymbol{C}_{k}^{H} \boldsymbol{y}_{u} \\
=\sum_{u=0}^{U-1} \sum_{m \in \mathcal{C}_{k}}\left(\hat{h}_{\tilde{s}_{k 1, m}}^{(u)}\right)^{*} C_{k m}^{*} y_{u m},
\end{gathered}
$$

where $\mathcal{C}_{k}$ is a set defined to contain all the $d_{x}$ indices having non-zero entries in $\boldsymbol{c}_{k}$. In (5), the second term can be simplified to

$$
\begin{aligned}
\tilde{s}_{k 2}^{*} \hat{\boldsymbol{h}}_{\tilde{s}_{k 1}}^{H}\left(\boldsymbol{I}_{U} \otimes \boldsymbol{C}_{k}^{H} \boldsymbol{C}_{i}\right) \hat{\boldsymbol{h}}_{\tilde{s}_{i 1}} \tilde{s}_{i 2} \\
\quad=\sum_{u=0}^{U-1} \sum_{m \in \mathcal{C}_{k} \cap \mathcal{C}_{i}} \tilde{s}_{k 2}^{*} C_{k m}^{*} C_{i m} \hat{h}_{\tilde{s}_{k 1}, m}^{(u) *} \hat{h}_{\tilde{s}_{i 1}, m}^{(u)} \tilde{s}_{i 2}
\end{aligned}
$$

where the set $\mathcal{C}_{k} \cap \mathcal{C}_{i}$ represents the specific indices, where both $\boldsymbol{c}_{k}$ and $\boldsymbol{c}_{i}$ have non-zero entries. Upon applying the simplified results in (6) and (7) to (5), we obtain

$$
\begin{aligned}
\hat{\boldsymbol{x}} & =\arg \max _{\tilde{\boldsymbol{x}} \in \mathcal{S}^{K}}\left\{\sum_{k=1}^{K} \sum_{u=0}^{U-1} \sum_{m \in \mathcal{C}_{k}} \Re\left\{\tilde{s}_{k 2}^{*} \hat{h}_{\tilde{s}_{k 1}, m}^{(u) *} C_{k m}^{*} y_{u m}\right\}\right. \\
& \left.-\frac{1}{2} \sum_{k=1}^{K} \sum_{i=1}^{K} \sum_{u=0}^{U-1} \sum_{m \in \mathcal{C}_{k} \cap \mathcal{C}_{i}} C_{k m}^{*} C_{i m} \tilde{s}_{k 2}^{*} \hat{h}_{\tilde{s}_{k 1}, m}^{(u) *} \hat{h}_{\tilde{s}_{i 1}, m}^{(u)} \tilde{s}_{i 2}\right\} .
\end{aligned}
$$

Observe from (8) that for a given $\hat{x}$ and assuming that two users share at most one subcarrier, the number of complex multiplications is about $\left(3 d_{x} K U+5 K^{2} U\right)$, which is much lower than the value of $\left(3 N K U+5 N K^{2} U\right)$ required, when full-weight spreading sequences are employed. Nevertheless, the complexity order of the MLD is still $\mathcal{O}\left(M^{K}\right)$, given the $M^{K}$ number of tests given by $\tilde{x} \in \mathcal{S}^{K}$ in (8). Hence for the massive number of connections supported by a SM/MCSCDMA system, this high complexity will prevent the MLD from practical implementation. Below we consider the symbolbased MAPD, which facilitates the implementation using belief propagation algorithms $[9,10,45]$.

\section{B. Maximum A Posteriori Detection (MAPD)}

Given a user, the MAPD operated on a symbol-by-symbol basis maximizes the a posteriori probability of a symbol [46], for estimating the symbol transmitted by the user. In detail, 
given the observations of (4), the estimate of the $k$ th user's symbol is obtained by solving the optimization problem of

$$
\hat{x}_{k}=\arg \max _{\tilde{x}_{k} \in \mathcal{S}}\left\{P\left(\tilde{x}_{k} \mid \boldsymbol{y}\right)\right\}, k=1,2, \cdots, K .
$$

Upon applying Bayes' rule [47], we obtain

$$
\begin{aligned}
\hat{x}_{k} & =\arg \max _{\tilde{x}_{k} \in \mathcal{S}} p\left(\boldsymbol{y} \mid \tilde{x}_{k}\right) P\left(\tilde{x}_{k}\right) \\
& =\arg \max _{\tilde{x}_{k} \in \mathcal{S}}\left\{\sum_{\boldsymbol{x}_{k} \in \mathcal{S}^{K-1}} P\left(\tilde{x}_{k}\right) P\left(\boldsymbol{x}_{k}\right) p\left(\boldsymbol{y} \mid \boldsymbol{x}_{k}, \tilde{x}_{k}\right)\right\},
\end{aligned}
$$

where $p\left(\boldsymbol{y} \mid \tilde{x}_{k}\right)$ is the probability density function (PDF) of $\boldsymbol{y}$ for a given $\tilde{x}_{k}$, while $p\left(\boldsymbol{y} \mid \boldsymbol{x}_{k}, \tilde{x}_{k}\right)$ is the PDF of $\boldsymbol{y}$ for a given $\left(\boldsymbol{x}_{k}, \tilde{x}_{k}\right)$. Here $\boldsymbol{x}_{i}$ is a $(K-1)$-length vector obtained from $\boldsymbol{x}$ after removing the $k$ th user's symbol, while $P\left(\tilde{x}_{k}\right)$ and $P\left(x_{k}\right)$ are the a priori probabilities of $\tilde{x}_{k}$ and $x_{k}$, respectively. It can be shown that for a given $\left(\boldsymbol{x}_{k}, \tilde{x}_{k}\right)$, the observations $\left\{y_{u n}\right\}$ are independent. Hence, we can further express (10) as

$$
\begin{aligned}
\hat{x}_{k}= & \arg \max _{\tilde{x}_{k} \in \mathcal{S}}\left\{\sum_{\boldsymbol{x}_{k} \in \mathcal{S}\left|\mathcal{K}_{k}\right|} P\left(\tilde{x}_{k}\right) P\left(\boldsymbol{x}_{k}\right)\right. \\
& \left.\times \prod_{u=0}^{U-1} \prod_{n=0}^{N-1} p\left(y_{u n} \mid \boldsymbol{x}_{k}, \tilde{x}_{k}\right)\right\},
\end{aligned}
$$

where $\mathcal{K}_{k}$ is the set of users interfering with user $k$. To be more specific, the set $\mathcal{K}_{k}$ contains all the indices of the users sharing at least one subcarrier with user $k$. Correspondingly, $\left|\mathcal{K}_{k}\right|$ is the cardinality of $\mathcal{K}_{k}$.

Let $\boldsymbol{x}_{[n]}$ be a $d_{c}$-length vector containing the symbols sent by the $d_{c}$ users sharing the $n$th subcarrier, which includes $\tilde{x}_{k}$, if user $k$ occupies the $n$th subcarrier. Then (11) can be further simplified to

$\hat{x}_{k}=\arg \max _{\tilde{x}_{k} \in \mathcal{S}}\left\{\sum_{\boldsymbol{x}_{k} \in \mathcal{S}\left|\mathcal{K}_{k}\right|} P\left(\tilde{x}_{k}\right) P\left(\boldsymbol{x}_{k}\right) \prod_{u=0}^{U-1} \prod_{n \in \mathcal{C}_{k}} p\left(y_{u n} \mid \boldsymbol{x}_{[n]}\right)\right\}$

When $\boldsymbol{x}_{[n]}$ is given, the PDF of $p\left(y_{u n} \mid \boldsymbol{x}_{[n]}\right)$ can be expressed as

$P\left(y_{u n} \mid \boldsymbol{x}_{[n]}\right)=\frac{1}{2 \pi \sigma^{2}} \exp \left(-\frac{\left\|y_{u n}-\sum_{i \in \mathcal{D}_{n}} \hat{h}_{s_{i 1}}^{(u)} s_{i 2} c_{i n}\right\|^{2}}{2 \sigma^{2}}\right)$,

where we express $\mathcal{D}_{n}$ as the set containing the indices of the users sharing the $n$th subcarrier.

We can infer from (12) that the complexity of the MAPD is $\mathcal{O}\left(M^{\left|\mathcal{K}_{k}\right|}\right)$. When sparse spreading codes are employed, $\left|\mathcal{K}_{k}\right|$ is determined by $d_{x}$ and $d_{c}$. More specifically, when regular sparse codes are employed, resulting in $d_{x}$ and $d_{c}$ being constants, we can readily show that we have $\left|\mathcal{K}_{k}\right|=d_{x}\left(d_{c}-1\right)$, provided that no two users share more than one subcarrier. Since we have $d_{x} \ll N$ and $d_{c} \ll K$, and typically $d_{x}=2,3$ and $d_{c}=3,4$ for SM/MC-SCDMA systems, the detection complexity of MAPD can be much lower than that of the MLD discussed in Section III-A. Furthermore, as shown below, with the aid of the MPA, a MPAD having a further reduced detection complexity, yet attaining a near-MAPD performance can be implemented.

\section{Message Passing Algorithm-Aided Detection (MPAD)}

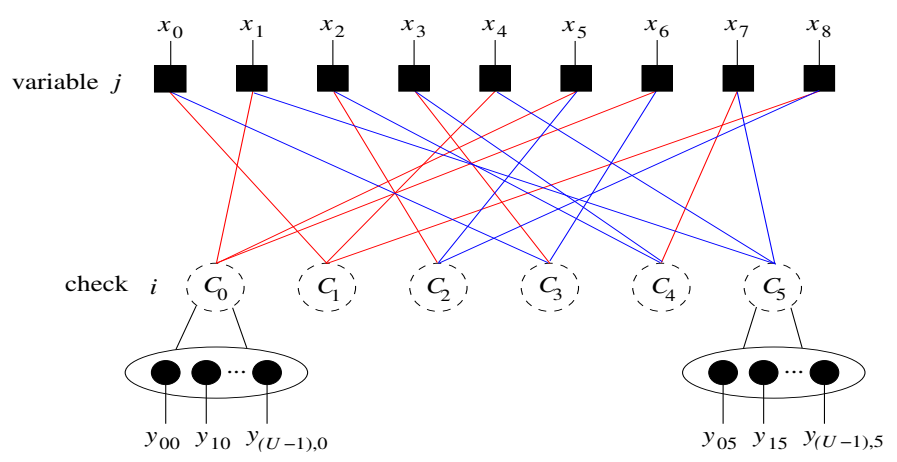

Fig. 3. A example showing the factor graph representation of the the SM/MCSCDMA system with regular sparse sequences and the parameters of $N=$ $6, K=9, d_{x}=2$, and $d_{c}=3$.

The classic factor graph $[1,9]$ that is convenient for characterizing the decoding of LDPC codes can be introduced to visualize the relationship between the $K$ users' transmitted symbols and the observations obtained at the BS receiver in the proposed SM/MC-SCDMA system. As shown in Fig. 3 for the case of $K=9$ and $N=6$, the $K$ symbols each taking a value from $\mathcal{S}$ are represented by the $K$ variable nodes, whereas the $U N$ observations obtained from $N$ subcarriers and $U$ RAs are represented by $N$ check nodes. Each check node corresponds to a subcarrier and contains $U$ observations obtained from the same subcarrier and $U$ RAs. As shown in Fig. 3, each check node is connected to a small number of variable nodes, which is determined by the sparse spreading codes assigned to the $K$ users. On the other hand, each variable node is connected to a small fraction of check nodes, which is determined by the number of non-zero elements of the sparse spreading code assigned to the corresponding user. As shown in Fig. 3, each variable node is connected to two check nodes, representing that any sparse spreading code has two non-zero elements. By contrast, each check node is connected to three users, meaning that each subcarrier is shared by three users.

For describing the MPAD, let the $d_{c}$ connections with the check node $i$, and the $d_{x}$ connections with the variable node $j$ be defined respectively by the sets

$$
\begin{aligned}
& \overline{\mathcal{C}}_{i}=\left\{j: 1 \leq j \leq K, e_{j i} \neq 0\right\}, i=0, \ldots, N-1 \\
& \overline{\mathcal{X}}_{j}=\left\{i: 0 \leq i \leq N-1, e_{j i} \neq 0\right\}, j=0, \ldots, K-1,
\end{aligned}
$$

where $e_{j i} \neq 0$ indicates that there exists an edge $e_{j i}$ between the check node $i$ and the variable node $j$. Based on the factor graph of Fig. 3, let us now detail the MPAD's operation in our proposed SM/MC-SCDMA system.

In Fig. 3 , via the edge $e_{j i}$, information can be conveyed upward from the check node $i$ to the variable node $j$. Information can also be conveyed downward from the variable node $j$ to the check node $i$. Correspondingly, the probability $\delta_{i, j}^{s_{m}, t}$ defines the amount of information conveyed from the check node $i$ to the variable node $j$ during the $t$ th iteration. Here, $\delta_{i, j}^{s_{m}, t}$ represents the probability of $x_{j}=s_{m}$, when given the probabilities received by the check node $c_{i}$ from all the connected variable nodes, after excluding $x_{j}$. By contrast, 
the amount of information sent from the variable node $x_{j}$ to the check node $c_{i}$ in the $t$ th iteration is expressed as $\eta_{j, i}^{s_{m}, t}$. Similarly, $\eta_{j, i}^{s_{m}, t}$ is the probability of $x_{j}=s_{m}$, when given the probabilities received by the variable node $x_{j}$ from all the connected check nodes, after excluding $c_{i}$. With the aid of the above definitions, following [48], the MPAD relies on the following steps.

First, $\eta_{j, i}^{s_{m}, 0}$ is initialised to $1 / M$ for all $s_{m} \in \mathcal{S}$ and any $e_{j, i} \neq 0$. Then, at the $t$-th iteration, $\delta_{i, j}^{s_{m}, t}$ for $i \in \overline{\mathcal{X}}_{i}$ and $j \in \overline{\mathcal{C}}_{j}$ can be updated as

$$
\begin{aligned}
\delta_{i, j}^{s_{m}, t}= & \sum_{\boldsymbol{x}_{[i]} \in \mathcal{S}^{d_{c}-1}, x_{j}=s_{m}}\left(\prod_{x_{v} \in \boldsymbol{x}_{[i]} \backslash x_{j}} \eta_{j, i}^{x_{v}, t}\right) \\
& \times \prod_{u=0}^{U-1} p\left(y_{u i} \mid \boldsymbol{x}_{[i]}, x_{j}=s_{m}\right), \\
& m=0,1, \ldots, M-1,
\end{aligned}
$$

where $\prod_{x_{v} \in \boldsymbol{x}_{[i]} \backslash x_{j}} \eta_{j, i}^{x_{v}, t}$ is the a priori probability of a given $\boldsymbol{x}_{[i]}$ with $x_{j}=s_{m}$, while $p\left(y_{u i} \mid \boldsymbol{x}_{[i]}, x_{j}=s_{m}\right)$ is given by (13). Observe from (15) that the information conveyed by the check node $i$ to the variable node $j$ is the product of the information gleaned from all the other edges connected to the check node $i$. The total information conveyed to the variable node $j$ is the sum of the information arriving from all the check nodes connected to the variable node $j$.

Next, at the $(t+1)$ th iteration, the values $\delta_{i, j}^{s_{m}, t}$ obtained in the $t$ th iteration are used to update $\eta_{j, i}^{s_{m}, t+1}$ for $j \in \overline{\mathcal{X}}_{j}$ and $i \in \overline{\mathcal{C}}_{i}$ as follows

$$
\eta_{j, i}^{s_{m}, t+1}=\varepsilon_{j, i} \prod_{v \in \overline{\mathcal{X}}_{j} \backslash i} \delta_{v, j}^{s_{m}, t}, m=0,1, \ldots, M-1,
$$

where $\varepsilon_{j, i}$ is the normalisation factor to ensure that $\sum_{m=0}^{M-1} \eta_{j, i}^{s_{m}, t+1}=1$.

Finally, after the pre-set number of iterations is reached, the detector detects the transmitted symbol of the $k$ th user as

$$
x_{k}=\arg \max _{s_{m} \in \mathcal{S}} \prod_{v \in \overline{\mathcal{X}}_{k}} \delta_{v, k}^{s_{m}, t}, k=0,2, \ldots, K-1 .
$$

We can see from (15) that the computational complexity is primarily dominated by the upward information transition. The number of multiplications is determined by the size of $\boldsymbol{x}_{[i]}$, while the detection complexity is determined by the $M^{d_{c}-1}$ possibilities in $\mathcal{S}^{d_{c}-1}$ of (15). Hence, the complexity of the MPAD is $\mathcal{O}\left(M^{d_{c}-1}\right)$.

\section{Analysis of the Single-User Performance AND ITS DISCUSSION}

This section first analyses the error probability of the SM/MC-SCDMA system supporting a single user, which gives the performance upper-bound. Furthermore, as shown by the results of references $[42,49,50]$ and those illustrated in Section $\mathrm{V}$, the single user BER performance bound can actually be viewed as the approximate BER performance of the SM/MCSCDMA system with the optimum MUD or MPAD, when the system supports multiple users up to a loading factor of two, meaning that each subcarrier on average supports two users.
Then, from the formulas derived in the single-user case, we gain insight into the performance impact of sparse sequences, and hence into their design.

\section{A. Analysis of Single-User Average Bit Error Ratio}

According to the MLD of (8), when the SM/MC-SCDMA system supports a single user, we can represent the MLD as

$$
\begin{aligned}
\hat{x}= & \arg \max _{\widetilde{x} \in \mathcal{X}}\left\{\sum_{u=0}^{U-1} \sum_{m \in \mathcal{C}} \Re\left\{\tilde{s}_{2}^{*} \hat{h}_{\tilde{s}_{1}, m}^{(u) *} C_{m}^{*} y_{u m}\right\}\right. \\
& \left.-\frac{1}{2} \sum_{u=0}^{U-1} \sum_{m \in \mathcal{C}}\left\|\hat{h}_{\tilde{s}_{1}, m}^{(u)} \tilde{s}_{2} C_{m}\right\|^{2}\right\},
\end{aligned}
$$

where the index $k$ is dropped for notational simplicity. Therefore, a detection error occurs, when we should have at least one scenario of $\tilde{x}\left(=\tilde{s}_{1} \mid \tilde{s}_{2}\right) \neq x\left(=s_{1} \mid s_{2}\right)$ resulting in

$$
\begin{aligned}
& \sum_{u=0}^{U-1} \sum_{m \in \mathcal{C}} \Re\left\{\tilde{s}_{2}^{*} \hat{h}_{\tilde{s}_{1}, m}^{(u) *} C_{m}^{*} y_{u m}\right\}-\frac{1}{2} \sum_{u=0}^{U-1} \sum_{m \in \mathcal{C}}\left\|\hat{h}_{\tilde{s}_{1}, m}^{(u)} \tilde{s}_{2} C_{m}\right\|^{2} \\
& >\sum_{u=0}^{U-1} \sum_{m \in \mathcal{C}} \Re\left\{s_{2}^{*} \hat{h}_{s_{1}, m}^{(u) *} C_{m}^{*} y_{u m}\right\}-\frac{1}{2} \sum_{u=0}^{U-1} \sum_{m \in \mathcal{C}}\left\|\hat{h}_{s_{1}, m}^{(u)} s_{2} C_{m}\right\|^{2}
\end{aligned}
$$

After some simplifications, (19) can be expressed as

$$
\begin{aligned}
& \sum_{u=0}^{U-1} \sum_{m \in \mathcal{C}} \Re\left\{\left(\tilde{s}_{2}^{*} \hat{h}_{\tilde{s}_{1}, m}^{(u) *}-s_{2}^{*} \hat{h}_{s_{1}, m}^{(u) *}\right) C_{m}^{*} y_{u m}\right\} \\
& >\frac{1}{2} \sum_{u=0}^{U-1} \sum_{m \in \mathcal{C}}\left(\left\|\hat{h}_{\tilde{s}_{1}, m}^{(u)} \tilde{s}_{2} C_{m}\right\|^{2}-\left\|\hat{h}_{s_{1}, m}^{(u)} s_{2} C_{m}\right\|^{2}\right) .
\end{aligned}
$$

Then, upon substituting $\boldsymbol{y}_{n}$ of (3) along with $K=1$ into (20), we can express the erroneous detection event in matrix (vector) form as

$$
\Re\left\{\left(\boldsymbol{H} \boldsymbol{e}_{\tilde{s}_{1}} \tilde{s}_{2}-\boldsymbol{H} \boldsymbol{e}_{s_{1}} s_{2}\right)^{H} \boldsymbol{n}_{U}\right\}>\frac{1}{2}\left\|\boldsymbol{H} \boldsymbol{e}_{\tilde{s}_{1}} \tilde{s}_{2}-\boldsymbol{H} \boldsymbol{e}_{s_{1}} s_{2}\right\|^{2},
$$

where $\boldsymbol{n}_{U}=\left(\boldsymbol{I}_{U} \otimes \boldsymbol{C}\right)^{H} \boldsymbol{n}$ is a complex Gaussian distributed vector having the PDF of $\mathcal{C N}\left(0, \gamma^{-1} \boldsymbol{I}_{U}\right)$. Consequently, the pairwise error probability (PEP), when the transmitted $x$ is detected as $\tilde{x} \neq x$ is given by [28]

$$
P_{E P}(x \rightarrow \tilde{x})=E_{\boldsymbol{H}}\left[Q\left(\sqrt{\frac{\gamma}{2}\left\|\boldsymbol{H} \boldsymbol{e}_{\tilde{s}_{1}} \tilde{s}_{2}-\boldsymbol{H} \boldsymbol{e}_{s_{1}} s_{2}\right\|^{2}}\right)\right]
$$

where $Q(x)$ is defined as $Q(x)=(2 \pi)^{-1 / 2} \int_{x}^{\infty} e^{-t^{2} / 2} d t$, and $E_{\boldsymbol{H}}[\cdot]$ stands for the expectation with respect to the channels between the $K$ users and the BS, which affect the terms $\boldsymbol{H} \boldsymbol{e}_{\tilde{s}_{1}}$ and $\boldsymbol{H e}_{\tilde{s}_{2}}$ in (22). With the alternative representation of the Q-function [51] of $Q(x)=\pi^{-1} \int_{0}^{\pi / 2} \exp \left(-\frac{x^{2}}{2 \sin ^{2} \theta}\right) d \theta$, the PEP of (22) can be expressed as

$$
\begin{aligned}
& P_{E P}(x \rightarrow \tilde{x}) \\
& =E_{\boldsymbol{H}}\left[\frac{1}{\pi} \int_{0}^{\frac{\pi}{2}} \exp \left(-\frac{\gamma}{4 \sin ^{2} \theta}\left\|\boldsymbol{H} \boldsymbol{e}_{\tilde{s}_{1}} \tilde{s}_{2}-\boldsymbol{H} \boldsymbol{e}_{s_{1}} s_{2}\right\|^{2}\right) d \theta\right] \\
& =\frac{1}{\pi} \int_{0}^{\frac{\pi}{2}} \Phi_{\left\|\boldsymbol{H} \boldsymbol{e}_{\tilde{s}_{1}} \tilde{s}_{2}-\boldsymbol{H} \boldsymbol{e}_{s_{1}} s_{2}\right\|^{2}}\left(-\frac{\gamma}{4 \sin ^{2} \theta}\right) d \theta
\end{aligned}
$$




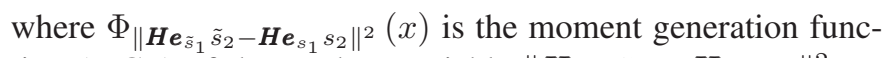
tion (MGF) of the random variable $\left\|\boldsymbol{H} \boldsymbol{e}_{\tilde{s}_{1}} \tilde{s}_{2}-\boldsymbol{H} \boldsymbol{e}_{s_{1}} s_{2}\right\|^{2}$.

Following the analysis in $[52,53]$, the average bit error ratio (ABER) of the single-user SM/MC-SCDMA system can be approximately evaluated from the union-bound as

$$
\begin{aligned}
\bar{P}_{b S} & \leq \frac{1}{M_{1} M_{2} b} \\
& \times \sum_{m_{1}=0}^{M_{1}-1} \sum_{m_{2}=0}^{M_{2}-1} \sum_{\tilde{m}_{1}=0}^{M_{1}-1} \sum_{\tilde{m}_{2}=0}^{M_{2}-1} D\left(\boldsymbol{b}_{1}^{\left(m_{1}\right)}\left|\boldsymbol{b}_{2}^{\left(m_{2}\right)}, \tilde{\boldsymbol{b}}_{1}^{\left(\tilde{m}_{1}\right)}\right| \tilde{\boldsymbol{b}}_{2}^{\left(\tilde{m}_{2}\right)}\right) \\
& \times P_{E P}\left(s_{1}^{\left(m_{1}\right)}\left|s_{2}^{\left(m_{2}\right)} \rightarrow \tilde{s}_{1}^{\left(\tilde{m}_{1}\right)}\right| \tilde{s}_{2}^{\left(\tilde{m}_{2}\right)}\right)
\end{aligned}
$$

where $D(\cdot, \cdot)$ is the Hamming distance between two binary entries, and $\boldsymbol{b}_{i}^{(m)}, s_{i}^{(m)}$ give the binary representation and symbol of the $m$-th SSK symbol (when $i=1$ ) or of the $m$-th APM symbol (when $i=2$ ). Furthermore, in (24), $s_{i}^{\left(m_{i}\right)} \rightarrow \tilde{s}_{i}^{\left(\tilde{m}_{i}\right)}$ means that the erroneous event takes the transmitted $s_{i}^{\left(m_{i}\right)}$ into detected $\tilde{s}_{i}^{\left(\tilde{m}_{i}\right)}$. As shown in [52], errors may corrupt only the SSK symbol, only the APM symbol, or both of them. When considering those three cases separately, the corresponding $P_{E P}(x \rightarrow \tilde{x})$ of (23a) can be derived as follows.

Erroneous SSK Symbol Only: When only the SSK symbol is in error, we have $\tilde{s}_{1} \neq s_{1}$ and $\tilde{s}_{2}=s_{2}$. In this case, (23a) can be reduced to

$$
\begin{aligned}
& P_{E P 1}\left(s_{1}\left|s_{2} \rightarrow \tilde{s}_{1}\right| s_{2}\right) \\
& =E_{\boldsymbol{H}}\left[\frac{1}{\pi} \int_{0}^{\frac{\pi}{2}} \exp \left(-\frac{\gamma\left|s_{2}\right|^{2}}{4 \sin ^{2} \theta}\left\|\boldsymbol{H} \boldsymbol{e}_{\tilde{s}_{1}}-\boldsymbol{H} \boldsymbol{e}_{s_{1}}\right\|^{2}\right) d \theta\right] \\
& =E_{\boldsymbol{H}}\left[\frac{1}{\pi} \int_{0}^{\frac{\pi}{2}} \exp \left(-\frac{\gamma\left|s_{2}\right|^{2}}{4 \sin ^{2} \theta}\left\|\boldsymbol{H} \boldsymbol{v}_{1}\right\|^{2}\right) d \theta\right] \\
& =\frac{1}{\pi} \int_{0}^{\frac{\pi}{2}} \Phi_{\left\|\boldsymbol{H} \boldsymbol{v}_{1}\right\|^{2}}\left(-\frac{\left|s_{2}\right|^{2} \gamma}{4 \sin ^{2} \theta}\right) d \theta
\end{aligned}
$$

where $\boldsymbol{v}_{1}=\boldsymbol{e}_{\tilde{s}_{1}}-\boldsymbol{e}_{s_{1}}$.

Erroneous APM Symbol Only: When errors only corrupt the APM symbol, we have $\tilde{s}_{2} \neq s_{2}$ and $\tilde{s}_{1}=s_{1}$. In this case, (23a) can be reduced to

$$
\begin{aligned}
& P_{E P 2}\left(s_{1}\left|s_{2} \rightarrow s_{1}\right| \tilde{s}_{2}\right) \\
& =E_{\boldsymbol{H}}\left[\frac{1}{\pi} \int_{0}^{\frac{\pi}{2}} \exp \left(-\frac{\gamma}{4 \sin ^{2} \theta}\left\|\boldsymbol{H} \boldsymbol{e}_{s_{1}} \tilde{s}_{2}-\boldsymbol{H} \boldsymbol{e}_{s_{1}} s_{2}\right\|^{2}\right) d \theta\right] \\
& =E_{\boldsymbol{H}}\left[\frac{1}{\pi} \int_{0}^{\frac{\pi}{2}} \exp \left(-\frac{\left|\tilde{s}_{2}-s_{2}\right|^{2} \gamma}{4 \sin ^{2} \theta}\left\|\boldsymbol{H} \boldsymbol{e}_{s_{1}}\right\|^{2}\right) d \theta\right] \\
& =\frac{1}{\pi} \int_{0}^{\frac{\pi}{2}} \Phi_{\left\|\boldsymbol{H} \boldsymbol{v}_{2}\right\|^{2}}\left(-\frac{\left|\tilde{s}_{2}-s_{2}\right|^{2} \gamma}{4 \sin ^{2} \theta}\right) d \theta
\end{aligned}
$$

where $\boldsymbol{v}_{2}=\boldsymbol{e}_{s_{1}}$.

Erroneous SSK and QAM Symbols: Finally, when errors occur simultaneously in both the SSK symbol and APM symbol, we have $\tilde{s}_{1} \neq s_{1}$ and $\tilde{s}_{2} \neq s_{2}$. Correspondingly, (23a) can be modified to

$$
\begin{aligned}
& P_{E P 3}\left(s_{1}\left|s_{2} \rightarrow \tilde{s}_{1}\right| \tilde{s}_{2}\right) \\
& =E_{\boldsymbol{H}}\left[\frac{1}{\pi} \int_{0}^{\frac{\pi}{2}} \exp \left(-\frac{\gamma}{4 \sin ^{2} \theta}\left\|\boldsymbol{H} \boldsymbol{e}_{\tilde{s}_{1}} \tilde{s}_{2}-\boldsymbol{H} \boldsymbol{e}_{s_{1}} s_{2}\right\|^{2}\right) d \theta\right] \\
& =E_{\boldsymbol{H}}\left[\frac{1}{\pi} \int_{0}^{\frac{\pi}{2}} \exp \left(-\frac{\gamma}{4 \sin ^{2} \theta}\left\|\boldsymbol{H} \boldsymbol{v}_{3}\right\|^{2}\right) d \theta\right] \\
& =\frac{1}{\pi} \int_{0}^{\frac{\pi}{2}} \Phi_{\left\|\boldsymbol{H} \boldsymbol{v}_{3}\right\|^{2}}\left(-\frac{\gamma}{4 \sin ^{2} \theta}\right) d \theta
\end{aligned}
$$

where $\boldsymbol{v}_{3}=\boldsymbol{e}_{\tilde{s}_{1}} \tilde{s}_{2}-\boldsymbol{e}_{s_{1}} s_{2}$.

As shown in (25), (26) and (27), in order to evaluate the ABER of (24), we have to derive the MGFs of $\Phi_{\left\|\boldsymbol{H} \boldsymbol{v}_{i}\right\|^{2}}(t)$ for $i=1,2$ and 3 . According to $[54,55]$, we know that

$$
\begin{aligned}
\left\|\boldsymbol{H} \boldsymbol{v}_{i}\right\|^{2} & =\sum_{n=0}^{U N-1} \boldsymbol{H}(n) \boldsymbol{v}_{i} \boldsymbol{v}_{i}^{H} \boldsymbol{H}^{H}(n) \\
& =\boldsymbol{h}^{T}\left[\boldsymbol{I}_{U N} \otimes\left(\boldsymbol{v}_{i} \boldsymbol{v}_{i}^{H}\right)\right] \boldsymbol{h}^{*},
\end{aligned}
$$

where $\boldsymbol{H}(n)$ represents the $n$th row of $\boldsymbol{H}$, and $\boldsymbol{h}=\operatorname{vec}\left(\boldsymbol{H}^{T}\right)$ is a $M_{1} U N$-length vector obtained from the rows of $\boldsymbol{H}$. Since the channels experience correlated Rayleigh fading, the MGF of $\left\|\boldsymbol{H} \boldsymbol{v}_{i}\right\|^{2}$ can be derived in the same way as that in $[54,55]$, which can be expressed as

$$
\Phi_{\left\|\boldsymbol{H} \boldsymbol{v}_{i}\right\|^{2}}(t)=\operatorname{det}\left[\boldsymbol{I}_{M_{1} U N}-t \boldsymbol{R}\left(\boldsymbol{I}_{U N} \otimes\left(\boldsymbol{v}_{i} \boldsymbol{v}_{i}^{H}\right)\right)\right]^{-1},
$$

where $\operatorname{det}(\boldsymbol{A})$ denotes the determinant of matrix $\boldsymbol{A}, \boldsymbol{R}$ is the covariance matrix of $h$ that can be expressed as

$$
\boldsymbol{R}=\left[\begin{array}{ccc}
\sqrt{\rho_{00}} & \cdots & \sqrt{\rho_{0\left(M_{1} U N-1\right)}} \\
\sqrt{\rho_{10}} & \cdots & \sqrt{\rho_{1\left(M_{1} U N-1\right)}} \\
\vdots & \ddots & \vdots \\
\sqrt{\rho_{\left(M_{1} U N-1\right) 0}} & \cdots & \sqrt{\rho_{\left(M_{1} U N-1\right)\left(M_{1} U N-1\right)}}
\end{array}\right]
$$

In $R, \rho_{m n}\left(m=0,1, \ldots, M_{1} U N-1 ; n=0,1, \ldots, M_{1} U N-\right.$ 1) can be obtained as follows. Let us express $m=M_{1} i+a$ and $n=M_{1} j+b$, where $i, j \in[0, N U-1]$ and $a, b \in\left[0, M_{1}-1\right]$. Furthermore, let $i=\left\lfloor\frac{i}{N}\right\rfloor N+c$ and $j=\left\lfloor\frac{j}{N}\right\rfloor N+d$, where $\lfloor x\rfloor$ gives the largest integer not exceeding $x$, while $c$ and $d$ are the two subcarrier indices derived from $i$ and $j$, respectively. Then, we can see that the indices $m$ and $n$ correspond to the same TA and the same RA, only when $j=\left\lfloor\frac{i}{N}\right\rfloor N,\left\lfloor\frac{i}{N}\right\rfloor N+1, \ldots,\left\lfloor\frac{i}{N}\right\rfloor N+N-1$ and $a=b$. Furthermore, $\rho_{m n}$ only becomes nonzero when the two subcarriers determined by $i$ and $j$, i.e., subcarriers $c$ and $d$, are activated by the spreading code. Based on the above analysis, we can show that the normalized correlation coefficient $\rho_{m n}(m, n)$ can be expressed in (31) [56]. From the above analysis and (31), we know that $R$ is a sparse matrix having at most $U M_{1} d_{x}^{2}$ and at least $U M_{1} d_{x}$ nonzero elements, corresponding to the pair of cases, when the $d_{x}$ subcarrier channels between a TA and a RA are correlated and independent, respectively. Note that, $\rho_{m m}$ on the diagonal of $\boldsymbol{R}$ is either zero, when the corresponding subcarrier is not used by the user, or one, if the user activates the corresponding subcarrier. Furthermore, if the $d_{x}$ subcarriers activated by the user experience independent 


$$
\rho_{m n}= \begin{cases}\frac{1}{L}+\frac{1}{L^{2}} \sum_{u=0}^{L-1} \sum_{v=0, v \neq u}^{L-1} \cos \left(\frac{2 \pi(c-d)(u-v)}{N}\right), & \text { if } m=M_{1} i+a, n=M_{1} j+b ; \\ & i=\left\lfloor\frac{i}{N}\right\rfloor N+c, j=\left\lfloor\frac{j}{N}\right\rfloor N+d \text { satisfy } \\ & a=b, c \in \mathcal{C}, d \in \mathcal{C}, \text { and } \\ & j=\left\lfloor\frac{i}{N}\right\rfloor N, \ldots, 2\left\lfloor\frac{i}{N}\right\rfloor N-1, \\ & \text { otherwise. }\end{cases}
$$

fading, the $U M_{1} d_{x}$ number of 1's are all on the diagonal of $R$.

Given the above preparation, we can now express (25), (26) and(27) respectively as

$$
\begin{aligned}
& P_{E P 1}\left(s_{1}\left|s_{2} \rightarrow \tilde{s}_{1}\right| s_{2}\right) \\
& =\frac{1}{\pi} \int_{0}^{\frac{\pi}{2}} \operatorname{det}\left(\boldsymbol{I}_{M_{1} U N}+\frac{\left|s_{2}\right|^{2} \gamma}{4 \sin ^{2} \theta} \boldsymbol{R}\left(\boldsymbol{I}_{U N} \otimes\left(\boldsymbol{v}_{1} \boldsymbol{v}_{1}^{H}\right)\right)\right)^{-1} d \theta
\end{aligned}
$$

$$
\begin{aligned}
& P_{E P 2}\left(s_{1}\left|s_{2} \rightarrow s_{1}\right| \tilde{s}_{2}\right) \\
& =\frac{1}{\pi} \int_{0}^{\frac{\pi}{2}} \operatorname{det}\left(\boldsymbol{I}_{M_{1} U N}+\frac{\left|\tilde{s}_{2}-s_{2}\right|^{2} \gamma}{4 \sin ^{2} \theta} \boldsymbol{R}\left(\boldsymbol{I}_{U N} \otimes\left(\boldsymbol{v}_{2} \boldsymbol{v}_{2}^{H}\right)\right)\right)^{-1}
\end{aligned}
$$

$$
\begin{aligned}
& P_{E P 3}\left(s_{1}\left|s_{2} \rightarrow \tilde{s}_{1}\right| \tilde{s}_{2}\right) \\
& =\frac{1}{\pi} \int_{0}^{\frac{\pi}{2}} \operatorname{det}\left(\boldsymbol{I}_{M_{1} U N}+\frac{\gamma}{4 \sin ^{2} \theta} \boldsymbol{R}\left(\boldsymbol{I}_{U N} \otimes\left(\boldsymbol{v}_{3} \boldsymbol{v}_{3}^{H}\right)\right)\right)^{-1} d \theta .
\end{aligned}
$$

Let us express the nonzero eigenvalues of $\boldsymbol{R}\left[\boldsymbol{I}_{U N} \otimes\left(\boldsymbol{v}_{1} \boldsymbol{v}_{1}^{H}\right)\right] \quad$ as $\quad\left\{\lambda_{11}, \lambda_{12}, \ldots, \lambda_{1 G_{1}}\right\}, \quad$ that of $\boldsymbol{R}\left[\boldsymbol{I}_{U N} \otimes\left(\boldsymbol{v}_{2} \boldsymbol{v}_{2}^{H}\right)\right]$ as $\left\{\lambda_{21}, \lambda_{22}, \ldots, \lambda_{2 G_{2}}\right\}$, and that of $\boldsymbol{R}\left[\boldsymbol{I}_{U N} \otimes\left(\boldsymbol{v}_{3} \boldsymbol{v}_{3}^{H}\right)\right]$ as $\left\{\lambda_{31}, \lambda_{32}, \ldots, \lambda_{3 G_{3}}\right\}$. Then, we can express (32) as

$$
P_{E P 1}\left(s_{1}\left|s_{2} \rightarrow \tilde{s}_{1}\right| s_{2}\right)=\frac{1}{\pi} \int_{0}^{\frac{\pi}{2}} \prod_{i=1}^{G_{1}}\left(1+\frac{\lambda_{1 i}\left|s_{2}\right|^{2} \gamma}{4 \sin ^{2} \theta}\right)^{-1} d \theta
$$

$$
\begin{gathered}
P_{E P 2}\left(s_{1}\left|s_{2} \rightarrow s_{1}\right| \tilde{s}_{2}\right) \\
=\frac{1}{\pi} \int_{0}^{\frac{\pi}{2}} \prod_{i=1}^{G_{2}}\left(1+\frac{\lambda_{2 i}\left|\tilde{s}_{2}-s_{2}\right|^{2} \gamma}{4 \sin ^{2} \theta}\right)^{-1} d \theta \\
P_{E P 3}\left(s_{1}\left|s_{2} \rightarrow \tilde{s}_{1}\right| \tilde{s}_{2}\right)=\frac{1}{\pi} \int_{0}^{\frac{\pi}{2}} \prod_{i=1}^{G_{3}}\left(1+\frac{\lambda_{3 i} \gamma}{4 \sin ^{2} \theta}\right)^{-1} d \theta
\end{gathered}
$$

Observe from the above derivation that the computation of $\bar{P}_{b S}$ is independent of the $M_{1}$ SSK symbol $s_{1}$, as seen in (25), (26) and (27), where the impact of the $M_{1}$ SSK symbol $s_{1}$ is imposed by the multiplication of $\boldsymbol{H e}_{s_{1}}$ or $\boldsymbol{H} \boldsymbol{e}_{\tilde{s}_{1}}$. However, any of them represents the selection of a column from $\boldsymbol{H}$. Since all columns of $\boldsymbol{H}$ have the same statistical properties, the resultant MGFs of the different $M_{1}$ SSK symbols are the same.
Furthermore, according to [52], when a $M_{1}$ SSK symbol is in error, the average number of erroneous bits is $b_{1} M_{1} /\left[2\left(M_{1}-\right.\right.$ $1)$. By contrast, for the Gray encoded $M_{2} \mathrm{APM}$ symbols, a symbol error typically yields a single bit error. Consequently, after considering the three erroneous events, we can simplify the expression of (24) to

$$
\begin{aligned}
& \bar{P}_{b S} \leq \frac{M_{1} b_{1}}{2 M_{2} b} \sum_{m_{2}=0}^{M_{2}-1} P_{E P 1}\left(s_{1}\left|s_{2}^{\left(m_{2}\right)} \rightarrow \tilde{s}_{1}\right| s_{2}^{\left(m_{2}\right)}\right) \\
& +\frac{1}{M_{2} b} \sum_{m_{2}=0}^{M_{2}-1} \sum_{\tilde{m}_{2} \neq m_{2}}^{M_{2}-1} D\left(\boldsymbol{b}_{2}^{\left(m_{2}\right)}, \tilde{\boldsymbol{b}}_{2}^{\left(\tilde{m}_{2}\right)}\right) \\
& \quad \times P_{E P 2}\left(s_{1}\left|s_{2}^{\left(m_{2}\right)} \rightarrow s_{1}\right| \tilde{s}_{2}^{\left(\tilde{m}_{2}\right)}\right) \\
& +\frac{1}{M_{2} b} \sum_{m_{2}=0}^{M_{2}-1} \sum_{\tilde{m}_{2} \neq m_{2}}^{M_{2}-1}\left[\frac{b_{1} M_{1}}{2}+\left(M_{1}-1\right) D\left(\boldsymbol{b}_{2}^{\left(m_{2}\right)}, \tilde{\boldsymbol{b}}_{2}^{\left(\tilde{m}_{2}\right)}\right)\right] \\
& \quad \times P_{E P 3}\left(s_{1}^{\left(m_{1}\right)}\left|s_{2}^{\left(m_{2}\right)} \rightarrow \tilde{s}_{1}^{\left(\tilde{m}_{1}\right)}\right| \tilde{s}_{2}^{\left(\tilde{m}_{2}\right)}\right),
\end{aligned}
$$

where $P_{E P 1}(\cdot), P_{E P 2}(\cdot)$ and $P_{E P 3}(\cdot)$ are given by (33a), (33b), and (33c), respectively. Note that in (34), the factor $M_{1} b_{1} / 2 M_{2} b$ in the first term at the right-hand side is obtained by letting, $\sum_{m_{1}=0}^{M_{1}-1}=M_{1}, \sum_{\tilde{m}_{1}=0}^{M_{1}-1}=M_{1}$, in (24), due to the above-mentioned fact that $P_{b S}$ is independent of the $M_{1}$ SSK symbol and owing to applying the average BER of $b_{1} M_{1} /\left[2\left(M_{1}-1\right)\right]$ imposed by a $M_{1}$ SSK symbol error after exploiting the approximation of $M_{1}-1 \approx M_{1}$. The factor of $1 / M_{2} b$ in the second term of (34) is obtained according to the condition of $s_{1}=\tilde{s}_{1}$ and hence we have $\sum_{m_{1}=0}^{M_{1}-1} \sum_{\tilde{m}_{1}=0}^{M_{1}-1}=M_{1}$. Finally, as for the third term in (24), firstly, we have $\sum_{m_{1}=0}^{M_{1}-1}=M_{1}$ and $\sum_{\tilde{m}_{1}=0}^{M_{1}-1}=M_{1}$ because $s_{1} \neq \tilde{s}_{1}$. Secondly, because both the $M_{1}$ SSK and $M_{2}$ QAM symbols are in error, the average number of erroneous bits per symbol is $\frac{b_{1} M_{1}}{2\left(M_{1}-1\right)}+D\left(\boldsymbol{b}_{2}^{\left(m_{2}\right)}, \tilde{\boldsymbol{b}}_{2}^{\left(\tilde{m}_{2}\right)}\right)$. Upon substituting these results into the third term of (24), we arrive at the third term at the right-hand side of (34).

\section{B. Discussions}

From (32a) - (33c) we realize that given $d_{x}$ and the fading environment, the eigenvalues in (33a) - (33c) are different when different sparse codes are used. Hence this results in different ABER performance. In order to minimize the ABER, 
it is required to design sparse codes that maximize

$$
\begin{aligned}
& J_{1}=\prod_{i=1}^{G_{1}}\left(1+\frac{\lambda_{1 i}\left|s_{2}\right|^{2} \gamma}{4 \sin ^{2} \theta}\right) \\
& J_{2}=\prod_{i=1}^{G_{2}}\left(1+\frac{\lambda_{2 i}\left|\tilde{s}_{2}-s_{2}\right|^{2} \gamma}{4 \sin ^{2} \theta}\right) \\
& J_{3}=\prod_{i=1}^{G_{3}}\left(1+\frac{\lambda_{3 i} \gamma}{4 \sin ^{2} \theta}\right) .
\end{aligned}
$$

Therefore, the specific structure of sparse codes impacts the achievable performance of SM/MC-SCDMA systems.

When there is single user or when the number of users is small, which results in a factor graph that is not well connected, the $d_{x}$ nonzero elements of a user should be distributed as evenly as possible. This allows the $d_{x}$ subcarriers activated by a user to be evenly distributed, and hence the detector beneficially attains frequency diversity in frequencyselective fading channels. We infer from the above formulas that evenly distributed sparse codes results in higher values for $J_{1}, J_{2}$ or/and $J_{3}$ in (35).

When the system supports a relatively high number of users, the corresponding factor graph is usually well connected. In this case, the MPAD is capable of gleaning multiuser diversity from the information exchange between users. However, as our experiments in Section V show, when the fading channel's frequency-selectivity is relatively low, for example $L=2,3,4$, the specific structure of sparse codes may still have a significant impact both on the values of $J_{1}, J_{2}$ or/and $J_{3}$, as well as on the achievable BER performance of SM/MC-SCDMA systems.

Below we provide our simulation results along with the single-user BER bound for characterizing the achievable BER performance of SM/MC-SCDMA systems.

\section{Performance Results}

In this section, the BER performance of SM/MC-SCDMA systems is characterized as a function of the number of TA/RAs and the number of resolvable paths, as well as that of the modulation levels and system scale. The SM/MC-SCDMA systems having different user loads of up to $K / N=2$ are considered. Note that in our performance studies, the $M_{2}$ APMs employed are $M_{2}$ QAMs. For convenience, the parameters used for generating the results for the individual figures are detailed in the figures.

In Fig. 4, we consider a single-user SM/MC-SCDMA system having $N=12$ subcarriers, 4SSK and QPSK modulation to investigate the impact of the channel's frequency-selectivity on the BER performance, where the user is randomly occupied $d_{x}=2$ of the 12 subcarriers. Both simulation results and the single-user BER bounds are evaluated based on the expressions derived in Section IV. Explicitly, the analytical BER bounds converge to the simulated BER, as the SNR increases. More specifically, for $L=1$, the BER bound is nearly the same as the simulated BER, provided that the SNR per bit obeys $\gamma_{0}>15 \mathrm{~dB}$, when $U=1$, and $\gamma_{0}>8 \mathrm{~dB}$, when $U=2$. For $L>1$, we can observe that the BER bound

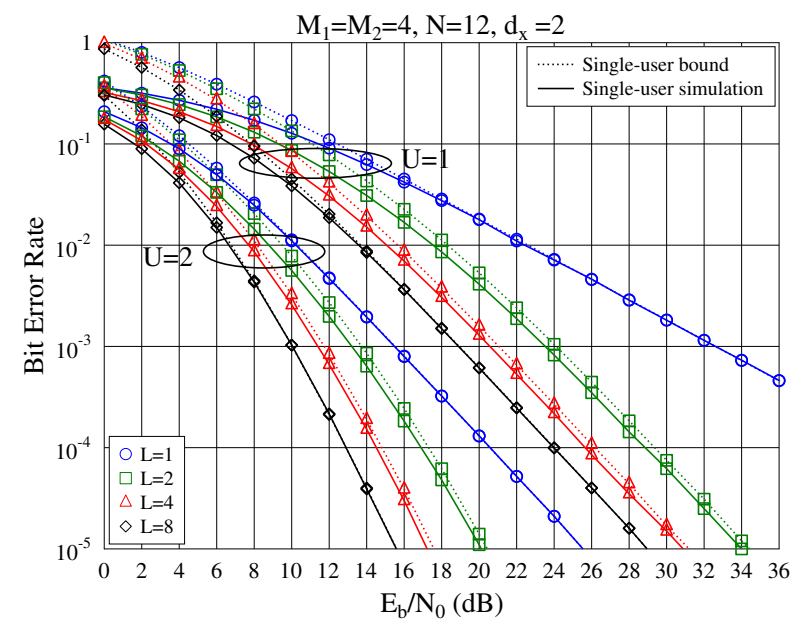

Fig. 4. Single-user BER bound of (34) for SM/MC-SCDMA systems with $N=12$ subcarriers communicating over frequency-selective Rayleigh fading channels having different number of paths.

approaches the simulated BER, as $L$ increases from 2 to 3 and to 4 . In general, the BER bound is tight, provided that the SNR is sufficiently high, resulting in a BER below 0.01. As shown in Fig. 4, the SM/MC-SCDMA system is capable of achieving both frequency diversity and space diversity, since the BER performance improves, as $L$ or $U$ increases.

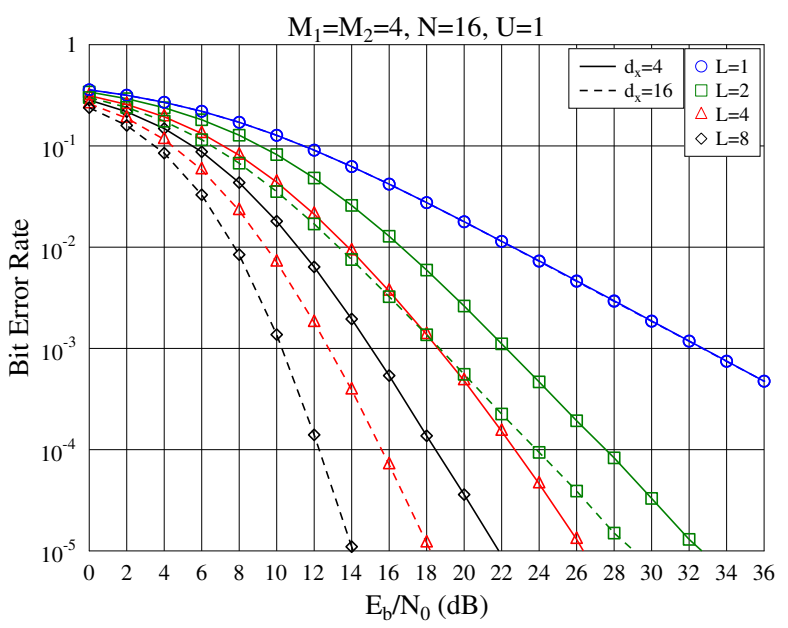

Fig. 5. Single-user BER performance of SM/MC-SCDMA systems, when a user occupies $d_{x}=4$ or all 16 subcarriers.

Fig. 5 demonstrates the impact of the number of subcarriers occupied by a user on the frequency diversity gain achieved by the SM/MC-SCDMA systems communicating over frequencyselective Rayleigh fading channels. For this investigation, we assume random sparse spreading sequences and set $U=$ $1, K=1$ as well as $d_{x}=4$ and 16 , respectively. Explicitly, in the case of $d_{x}=N=16, \mathrm{SM} / \mathrm{MC}$-SCDMA achieves full frequency-diversity, and its performance is hence always better than that of the corresponding SM/MC-SCDMA associated with $d_{x}=4$, provided that $L>1$. We should note that for the case of $d_{x}=4$, the achievable frequency-diversity gain 
is limited by both the values of $L$ and $d_{x}$. Hence, as shown in Fig. 5, when $L$ is relatively large, such as $L=4,8$, the loss of diversity gain due to the use of the sparse sequence of $d_{x}=4$ is significant in comparison to the case of $d_{x}=16$, which achieves full frequency diversity.

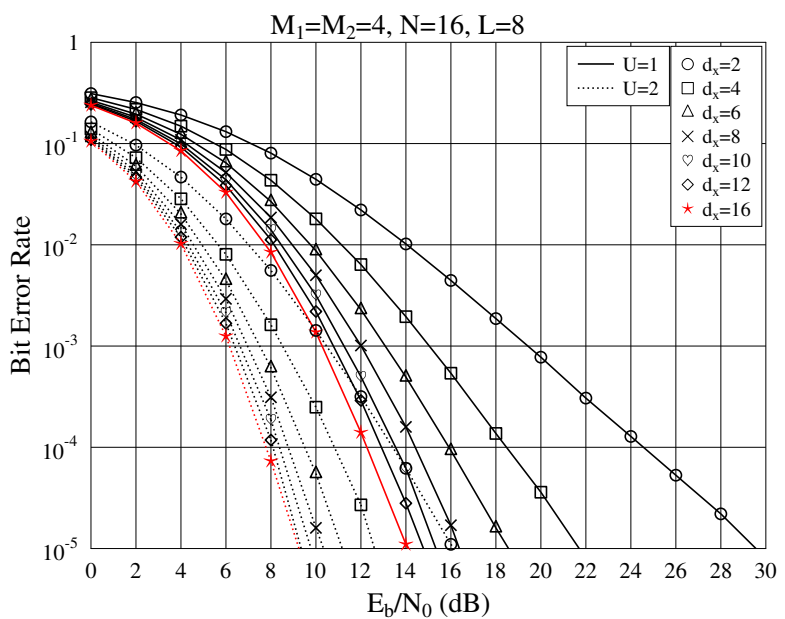

Fig. 6. Single-user BER performance of SM/MC-SCDMA systems, when a user occupies different number of subcarriers $\left(d_{x}\right)$ for information transmission.

The above observation is augmented in Fig. 6, where we fix $L=8$ and $U=1$ or 2 , but vary the value of $d_{x}$ in a singleuser SM/MC-SCDMA system communicating over frequencyselective Rayleigh fading channels. As expected, for a given value of $U$, the BER performance improves as $d_{x}$ increases, and exhibits a converging trend for $d_{x} \geq 6$. Therefore, from the results shown in Figs. 5 and 6 we infer that in the SM/MCSCDMA supporting single-user (or a low number of users), the employment of sparse sequences strikes a trade-off with the achievable frequency-diversity. Below we will illustrate that the structure of sparse sequences also has an impact on the BER performance of SM/MC-SCDMA systems.

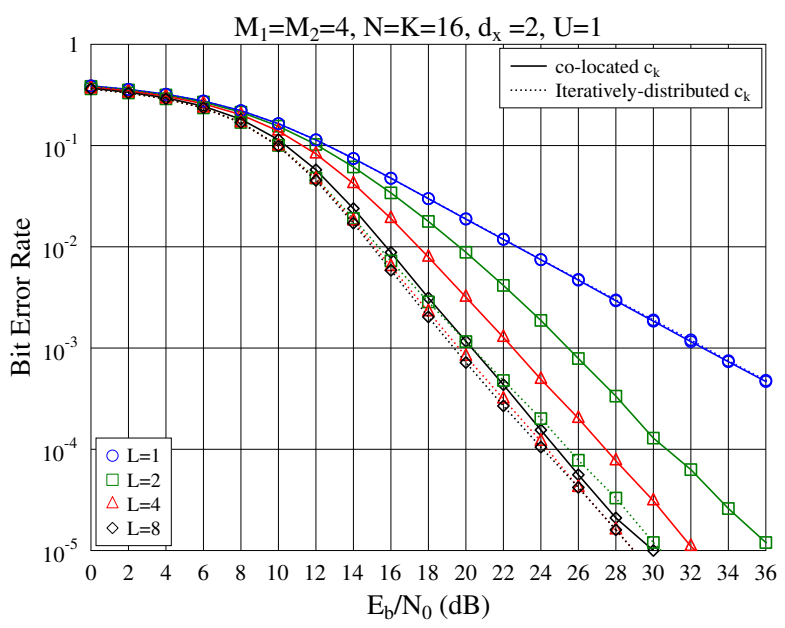

Fig. 7. Performance comparison of SM/MC-SCDMA systems, when the non-zero elements of users are co-located or iteratively distributed.
In Fig. 7, we demonstrate the effect of the sparse codes having different structures on the achievable BER performance. Specifically, we compare two classes of sparse codes, namely the co-located and iteratively distributed sparse codes. Given the parameters of $N=K=16, d_{x}=2$, and that two adjacent users share a single nonzero element, the nonzero elements in these classes of sparse codes are designed to be allocated as follows. For the co-located sparse codes, the $d_{x}=2$ nonzero elements of user $k, k=0,1, \ldots, 15$, are at the positions of $(k+l) \bmod N$ for $l=0,1$. For the iteratively distributed sparse codes, the $d_{x}=2$ nonzero elements of user $k=0,1, \ldots, 7$, are at the positions of $(k+8 l) \bmod 16$ for $l=0,1$, while the $d_{x}=2$ nonzero elements of user $k=8,9, \ldots, 15$, are at the positions of $(k+9 l) \bmod 16$ for $l=0,1$. Observe from Fig. 7 that while the co-located sparse codes allow us to obtain multiuser diversity gain via information exchange between different users, the frequency diversity gain achieved by employing iteratively distributed sparse codes is significant for a relatively small value of $L$, such as $L=2,4$. Specifically for $L=2$ and at the BER of $10^{-3}$, instead of the co-located sparse codes, employing the iteratively distributed sparse codes is capable of attaining an extra of $5.5 \mathrm{~dB}$ diversity gain. However, when the channel is highly frequency-selective, resulting in say $L=8$, the extra diversity gain provided by the iteratively distributed sparse codes is insignificant compared to that obtained by the colocated sparse codes.

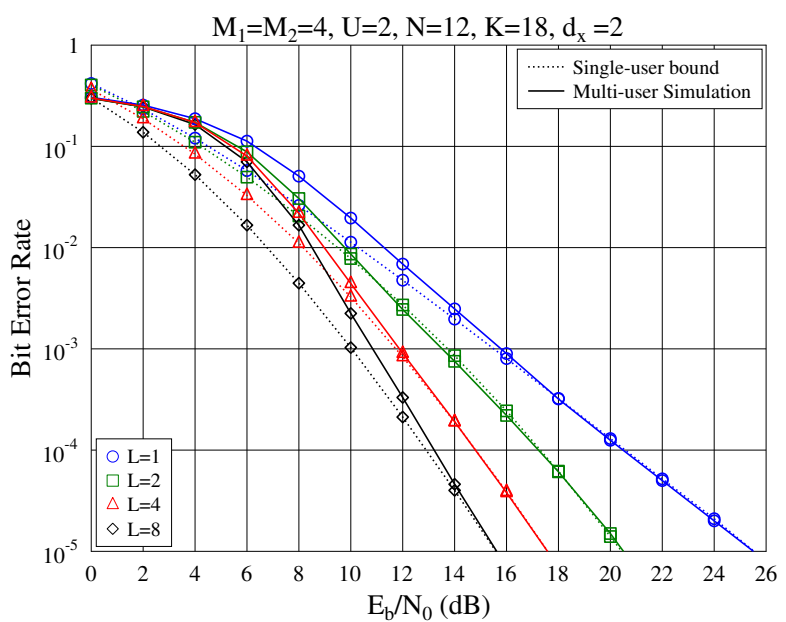

Fig. 8. BER performance of SM/MC-SCDMA systems employing $N=12$ subcarriers to support $K=18$ users.

In Fig. 8 the BER performance of the SM/MC-SCDMA systems supporting multiple users at a loading factor of $K / N=1.5$ is investigated for several values of $L$. Note that in Fig. 8 and in the ensuing figures, the single-user bound evaluated from (34) is also plotted. First, we can explicitly observe the benefits of diversity gain, as demonstrated by the improved BER performance upon increasing $L$. Second, due to the multiuser interference (MUI), the BER performance observed in the low SNR region degrades against the singleuser BER bound. However, when the SNR is sufficiently high to reduce the BER below $10^{-3}$ or $10^{-4}$, the MPAD allows 
the highly loaded SM/MC-SCDMA systems to achieve a BER performance near the single-user BER bound.

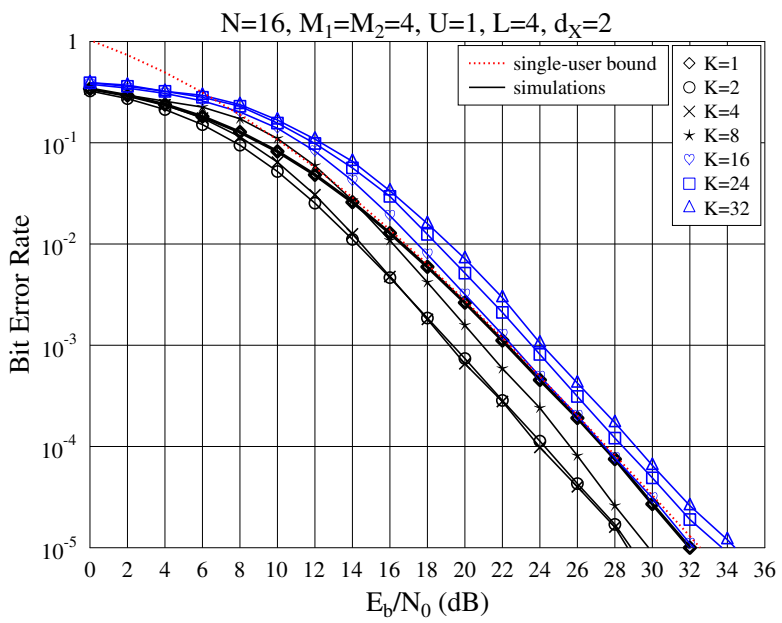

Fig. 9. BER performance of SM/MC-SCDMA system employing $N=16$ subcarriers to support different number of users.

By fixing $U=1, d_{x}=2, L=4$ and $N=16$, Fig. 9 depicts the BER of SM/MC-SCDMA systems supporting different number of users, with the loading factor up to $K / N=2$. Here the co-located sparse codes are employed. In addition to the 16 codes used in Fig. 7, an extra 16 codes having non-zero positions at $(k+l) \bmod N$ for $l=0,2$ are used, in order to support $K=32$ users. From the results we infer the following observation. First, the existence of multiple users has twin-fold effect. On the one hand, it generates MUI as in any non-orthogonal multiuser systems. On the other hand, it attains a beneficial diversity gain, namely, multiuser diversity. In more detail, when the system only supports a single user, the maximum diversity order that can be achieved by the system is fixed to $d_{x}=2$, since only $d_{x}=2$ subcarriers are activated and hence the information used for detecting the user can only come from the two active subcarriers. By contrast, when there are multiple users, then more than two subcarriers are active. In this case, a higher diversity order can be exploited for detecting a user by the MPA algorithm via the resultant beneficial information exchange between the different subcarriers. Consequently, as seen in Fig. 9, the BER performance first improves, as the number of users increases from 1 to 4 . However, as the number of users further increases, the MUI starts dominating the achievable performance, hence resulting in the degradation of the BER performance. Second, the results seen in Fig. 9 imply that the MPAD is nearoptimum, and even with the loading factor of 2 , there is no error-floor in the SNR range of interest. The performance of the SM/MC-SCDMA system is only about $5 \mathrm{~dB}$ worse than the best possible performance, which is achieved for $K=4$ users.

Fig. 10 quantifies the effect of the number of modulation levels for $M_{1} \mathrm{SSK}$ and $M_{2} \mathrm{QAM}$ on the BER performance of SM/MC-SCDMA systems employing regular sparse sequences and $d_{x}=2, d_{c}=3$. First, as shown in Fig. 10 for $M_{2} \mathrm{QAM}$ modulation, while a higher QAM order achieves a higher

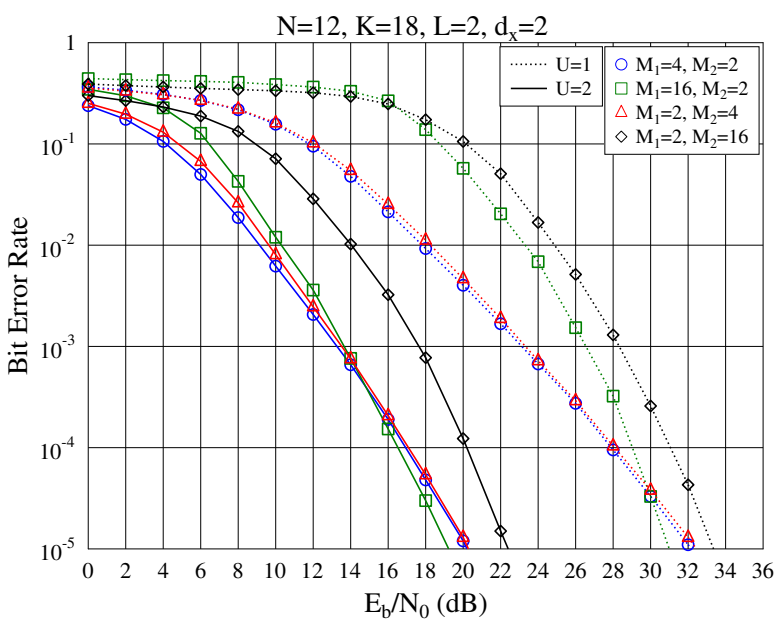

Fig. 10. BER performance of SM/MC-SCDMA systems employing $N=12$ subcarriers to support $K=18$ users, i.e., with a loading factor of 1.5.

bandwidth-efficiency, this is attained at the cost of a degraded BER performance [57]. However, the SM/MC-SCDMA system having the parameters of $M_{1}=16, M_{2}=2$ and $U=1$ is outperformed by the system having the parameters of $M_{1}=4$, $M_{2}=2$ and $U=1$.

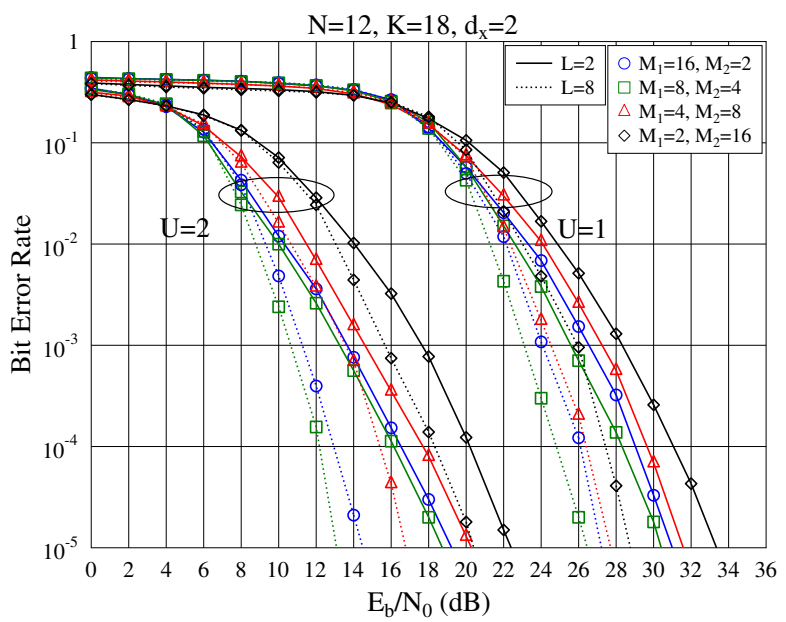

Fig. 11. BER performance of the SM/MC-SCDMA systems with a loading factor of 1.5 , when given $b=b_{1}+b_{2}=5$ BPS but using different modulation levels.

In Fig. 10, we have demonstrated that the SSK and APM schemes exhibit different characteristics in terms of energyand bandwidth-efficiency, as well as BER performance. Therefore, in Fig. 11 we further investigate the effect of bit allocation between the two modulation schemes on the system's error performance, under the constraint that the total number of BPS is given by $b=b_{1}+b_{2}=5$. The other settings for Fig. 11 are the same as those for Fig. 10. The results in Fig. 11 show that for all the cases defined by $U=1,2$ and $L=2,8$, the combination of 8 SSK with 4 QAM achieves the best BER among the four combinations considered. This observation implies that given the total number of BPS, there 
exists an optimum bit sharing between SSK and APM that could result in the best BER.

Note that, the results of Figs. 4, 10, and 11 also demonstrate that employing more RAs improves the system's BER, owing to the increase of space diversity.

In Fig. 12, we now compare our SM/MC-SCDMA system to several other systems, including the SM/MC-SCMA system, which is the generalization of our SM/MC-SCDMA arrangement, the MC-SCDMA system operating without spatial modulation, and an extended version of the MC-SCMA system [58]. Note that, when SCMA [58] is considered, the modulation, constellation shaping and spreading are intrinsically amalgamated for generating a codebook. In this way, user data are no longer carried purely by the classic APM symbols but by the specifically designed multi-dimensional codewords. In Fig. 12 we assume that the system employs $N=12$ subcarriers for supporting $K=18$ users and transmits at a rate of $b=b_{1}+b_{2}=4$ BPS per user. The SCMA codebook follows the design of [58], and the corresponding variable of $M_{2}=16$ represents the per user codebook size. As seen in Fig. 12, the spatial modulated schemes, namely the SM/MC-SCDMA and SM/MC-SCMA, achieve a better BER performance than the other two schemes operating without spatial modulation. Additionally, the SCMA-based scheme is capable of achieving about $1.5 \mathrm{~dB}$ gain over its corresponding SCDMA counterpart, which facilitates fair comparison with the SCMA and SCDMA schemes of [3]. Furthermore, for MPAD, the detection complexity of both SCDMA and of the corresponding SCMA is the same, since in both systems, factor graphs used by the MPA are the same. However, we should note that the transmitter of a SCMA system has higher complexity than that of the corresponding SCDMA system, where the extra complexity imposed by the codeword design, code allocation and storage. By contrast, in SCDMA systems, every user is only assigned a single code for spreading. Additionally, it it worth mentioning that the comparison of spatial modulated systems to conventional MIMO schemes was carried out in $[18,21,59]$, demonstrating that a conventional MIMO scheme is capable of achieving a better BER performance than the corresponding spatial modulated scheme, but typically imposes a higher detection complexity, requires more RF chains, strict inter-antenna synchronization, etc.

Finally, in Fig. 13 we depict the BER of the SM/MCSCDMA systems at different scales at a fixed loading factor of 2 , when communicating over frequency-selective fading channels, and assuming 2SSK/BPSK modulation. Firstly, the results show that the BER improves for $U=1$ and slightly also for $U=2$, as the SM/MC-SCDMA system dimension becomes larger. This observation follows the MIMO principles [60]: the capacity of MIMO channels increases at least linearly upon increasing the minimum of the number of TA, and RAs. As shown in Fig. 13, when comparing the scenario of $L=2, N=128, K=256$ to that of $L=2, N=16, K=$ 32 , where both cases attain the same bandwidth efficiency of 4 bits per subcarrier per symbol. However, the former scenario requires a $5 \mathrm{~dB}$ lower SNR at the BER of $10^{-4}$. By contrast, when comparing the scenario of $L=8, N=128, K=256$ to that of $L=8, N=16, K=32$, the former scheme

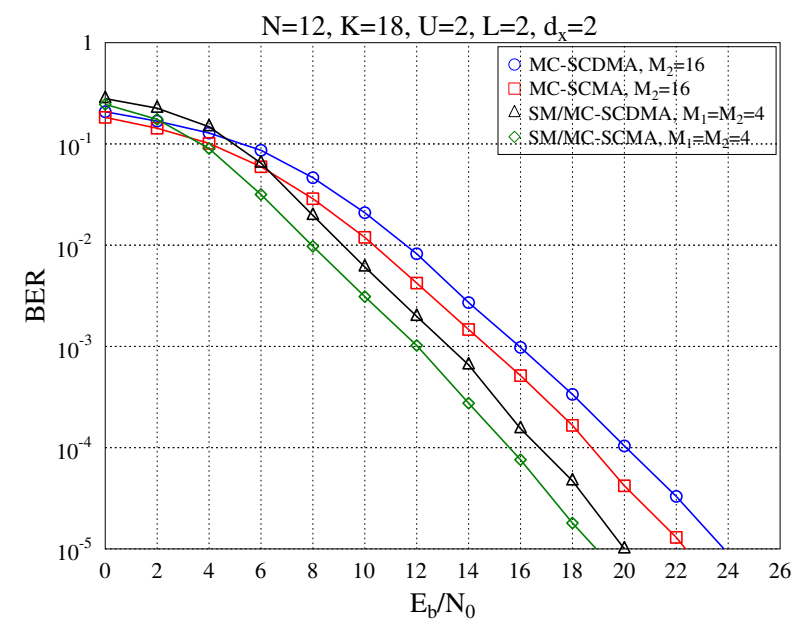

Fig. 12. Comparison of BER performance of the SM/MC-SCDMA, MCSCDMA, SM/MC-SCMA and MC-SCMA systems with a system load of $150 \%$.

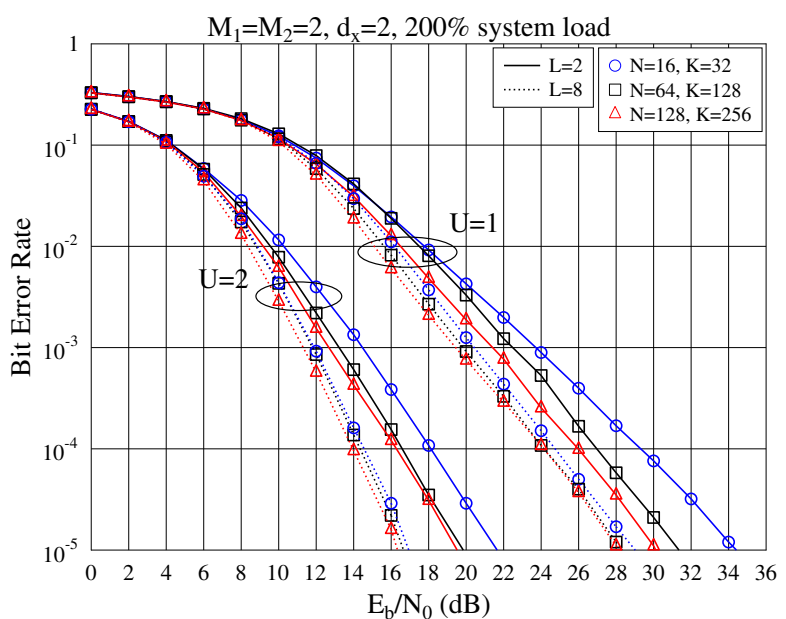

Fig. 13. Comparison of BER performance of the SM/MC-SCDMA systems employing different number of subcarriers to support a fixed system load of $200 \%$.

has a $1 \mathrm{~dB}$ SNR gain. Hence, although a more dispersive frequency-selective channel results in a lower SNR gain of $1 \mathrm{~dB}$, in general, the SM/MC-SCDMA system supported by the MPA detection becomes more efficient, as the system becomes larger. Secondly, benefiting from spreading and of MPA, a frequency diversity gain can be achieved, yielding a BER improvement, as the channel becomes more frequencyselective. Additionally, as also demonstrated in the previous figures, using more RAs improves the space diversity gain, hence resulting in significant performance improvement when increasing the number of RAs from $U=1$ to $U=2$.

\section{CONCLUSions}

A SM/MC-SCDMA scheme has been proposed with the motivation of providing massive connectivity in nextgeneration wireless systems. In SM/MC-SCDMA, the spatially modulated bits activate a fraction of TAs to transmit the APM 
modulated bits, which are transmitted after sparse spreading in the frequency-domain over frequency-selective Rayleigh fading channels with the aid of MC signaling. A single-user BER bound has been derived based on the MGF, which is used for investigating the effect of the codes' sparsity on the BER performance. In terms of the detection, MPAD has been introduced to implement low-complexity detection, while achieving near-MLD performance. Our results also demonstrate that in addition to frequency diversity, the proposed SM/MC-SCDMA system attains space diversity by employing RAs. We also observed that there exists an optimal bit-sharing between the SSK and APM modulations. We demonstrated that the BER performance only slightly degrades at a user load of $200 \%$, and the system performs best, when its dimension is large.

In summary, our SM/MC-SCDMA scheme has the following merits. With the aid of SM, MC signalling and low-complexity MPAD, our SM/MC-SCDMA scheme has a compellingly low complexity. Relying on SM, the transmitter of SM/MC-SCDMA can relax the inter-antenna synchronization specifications. Employing sparse spreading beneficially mitigates the PAPR problem of MC signalling at transmitter, while enjoying low-complexity MPAD at the receiver. With the advent of MC signalling and multiple RAs, the performance of SM/MC-SCDMA systems benefits from both space and frequency diversity. Lastly, the introduction of the low-complexity MPAD allows SM/MC-SCDMA systems to achieve near-MLD performance, even when the systems are heavily loaded with the loading factors as high as two or even higher. Owing to these merits, SM/MC-SCDMA constitutes a promising MA scheme for ultra-dense device-centric wireless systems requiring massive connectivity.

In this paper, we have compared the BER performance of our SM/MC-SCDMA and its generalized counterpart SM/MCSCMA, showing that an extra shaping gain is available. Hence, our future work may further investigate the code design, constellation shaping and in particular, the user/codeword scheduling both in SM/MC-SCDMA and SM/MC-SCMA systems. Additionally, attractive low complexity detectors should be designed for the SM/MC-SCDMA and SM/MC-SCMA systems.

\section{REFERENCES}

[1] R. Hoshyar, F. P. Wathan, and R. Tafazolli, "Novel low-density signature for synchronous CDMA systems over AWGN channel," IEEE Transactions on Signal Processing, vol. 56, no. 4, pp. 1616-1626, 2008.

[2] D. Guo and C.-C. Wang, "Multiuser detection of sparsely spread CDMA," IEEE Journal on Selected Areas in Communications, vol. 26, no. 3, pp. 421-431, 2008

[3] H. Nikopour and H. Baligh, "Sparse code multiple access," in 2013 IEEE 24th Annual International Symposium on Personal, Indoor and Mobile Radio Communications (PIMRC). IEEE, 2013, pp. 332-336.

[4] A. Osseiran, F. Boccardi, V. Braun, K. Kusume, P. Marsch, M. Maternia, O. Queseth, M. Schellmann, H. Schotten, H. Taoka et al., "Scenarios for $5 \mathrm{G}$ mobile and wireless communications: the vision of the METIS project," IEEE Communications Magazine, vol. 52, no. 5, pp. 26-35, 2014.

[5] J. Zhang, L. Lu, Y. Sun, Y. Chen, J. Liang, J. Liu, H. Yang, S. Xing, Y. Wu, J. Ma et al., "PoC of SCMA-based uplink grant-free transmission in UCNC for 5G," IEEE Journal on Selected Areas in Communications, vol. 35, no. 6, pp. 1353-1362, 2017.

[6] L. Dai, B. Wang, Z. Ding, Z. Wang, S. Chen, and L. Hanzo, "A survey of non-orthogonal multiple access for 5G," IEEE Communications Surveys \& Tutorials, vol. 20, no. 3, pp. 2294-2323, 2018.
[7] Y. Cai, Z. Qin, F. Cui, G. Y. Li, and J. A. McCann, "Modulation and multiple access for 5G networks," IEEE Communications Surveys \& Tutorials, vol. 20, no. 1, pp. 629-646, 2018.

[8] L. Hanzo, L.-L. Yang, E.-L. Kuan, and K. Yen, Single-and multi-carrier DS-CDMA: multi-user detection, space-time spreading, synchronisation, standards and networking. John Wiley \& Sons, 2003.

[9] F. R. Kschischang, B. J. Frey, and H.-A. Loeliger, "Factor graphs and the sum-product algorithm," IEEE Transactions on Information Theory, vol. 47, no. 2, pp. 498-519, 2001.

[10] F. R. Kschischang, "Codes defined on graphs," IEEE Communications Magazine, vol. 41, no. 8, pp. 118-125, 2003.

[11] H. Mu, Z. Ma, M. Alhaji, P. Fan, and D. Chen, "A fixed low complexity message pass algorithm detector for up-link SCMA system," IEEE Wireless Communications Letters, vol. 4, no. 6, pp. 585-588, 2015.

[12] A. Bayesteh, H. Nikopour, M. Taherzadeh, H. Baligh, and J. Ma, "Low complexity techniques for scma detection," in 2015 IEEE Globecom Workshops (GC Wkshps). IEEE, 2015, pp. 1-6.

[13] L. Yang, Y. Liu, and Y. Siu, "Low complexity message passing algorithm for scma system," IEEE Communications Letters, vol. 20, no. 12, pp. 2466-2469, 2016

[14] W. Yuan, N. Wu, Q. Guo, Y. Li, C. Xing, and J. Kuang, "Iterative receivers for downlink mimo-scma: Message passing and distributed cooperative detection," IEEE Transactions on Wireless Communications, vol. 17, no. 5, pp. 3444-3458, 2018.

[15] Y. Du, B. Dong, Z. Chen, X. Wang, and P. Gao, "Improved serial scheduling-based detection for sparse code multiple access systems," IEEE Wireless Communications Letters, vol. 6, no. 5, pp. 570-573, 2017.

[16] X. Ma, L. Yang, Z. Chen, and Y. Siu, "Low complexity detection based on dynamic factor graph for SCMA systems," IEEE Communications Letters, vol. 21, no. 12, pp. 2666-2669, 2017.

[17] R. Y. Mesleh, H. Haas, S. Sinanovic, C. W. Ahn, and S. Yun, "Spatial modulation," IEEE Transactions on Vehicular Technology, vol. 57, no. 4, pp. 2228-2241, 2008.

[18] M. Di Renzo, H. Haas, and P. M. Grant, "Spatial modulation for multiple-antenna wireless systems: A survey," IEEE Communications Magazine, vol. 49, no. 12, 2011.

[19] A. Younis, N. Serafimovski, R. Mesleh, and H. Haas, "Generalised spatial modulation," in Signals, Systems and Computers (ASILOMAR), 2010 Conference Record of the Forty Fourth Asilomar Conference on. IEEE, 2010, pp. 1498-1502.

[20] J. Wang, S. Jia, and J. Song, "Generalised spatial modulation system with multiple active transmit antennas and low complexity detection scheme," IEEE Transactions on Wireless Communications, vol. 11, no. 4, pp. 1605-1615, 2012.

[21] M. Di Renzo, H. Haas, A. Ghrayeb, S. Sugiura, and L. Hanzo, "Spatial modulation for generalized MIMO: Challenges, opportunities, and implementation," Proceedings of the IEEE, vol. 102, no. 1, pp. 56-103, 2014.

[22] P. Yang, M. Di Renzo, Y. Xiao, S. Li, and L. Hanzo, "Design guidelines for spatial modulation," IEEE Communications Surveys \& Tutorials, vol. 17 , no. 1 , pp. 6-26, 2015.

[23] I. A. Hemadeh, M. El-Hajjar, and L. Hanzo, "Hierarchical multifunctional layered spatial modulation," IEEE Access, vol. 6, pp. 94929533, 2018.

[24] L. Xiao, P. Xiao, Y. Xiao, C. Wu, H. V. Nguyen, I. A. Hemadeh, and L. Hanzo, "Transmit antenna combination optimization for generalized spatial modulation systems," IEEE Access, vol. 6, pp. 41 866-41882, 2018.

[25] Y. A. Chau and S.-H. Yu, "Space modulation on wireless fading channels," in Vehicular Technology Conference, 2001. VTC 2001 Fall. IEEE VTS 54th, vol. 3. IEEE, 2001, pp. 1668-1671.

[26] J. Jeganathan, A. Ghrayeb, L. Szczecinski, and A. Ceron, "Space shift keying modulation for MIMO channels," IEEE Transactions on Wireless Communications, vol. 8, no. 7, pp. 3692-3703, 2009.

[27] N. Serafimovski, S. Sinanović, M. Di Renzo, and H. Haas, "Multiple access spatial modulation," EURASIP Journal on Wireless Communications and Networking, vol. 2012, no. 1, p. 299, 2012.

[28] M. D. Renzo and H. Haas, "Bit error probability of space-shift keying MIMO over multiple-access independent fading channels," IEEE Transactions on Vehicular Technology, vol. 60, no. 8, pp. 3694-3711, Oct 2011.

[29] L.-L. Yang, "Signal detection in antenna-hopping space-division multiple-access systems with space-shift keying modulation," IEEE Transactions on Signal Processing, vol. 60, no. 1, pp. 351-366, 2012

[30] A. Garcia-Rodriguez and C. Masouros, "Low-complexity compressive sensing detection for spatial modulation in large-scale multiple access 
channels," IEEE Transactions on Communications, vol. 63, no. 7, pp. 2565-2579, July 2015 .

[31] J. Zheng, "Low-complexity detector for spatial modulation multiple access channels with a large number of receive antennas," IEEE Communications Letters, vol. 18, no. 11, pp. 2055-2058, Nov 2014.

[32] T. L. Narasimhan, P. Raviteja, and A. Chockalingam, "Generalized spatial modulation in large-scale multiuser MIMO systems," IEEE Transactions on Wireless Communications, vol. 14, no. 7, pp. 3764 3779, 2015.

[33] J. W. Kim, M. Irfan, M. A. Shayokh, and S. Y. Shin, "Selective non-orthogonal multiple access (NOMA) and spatial modulation (SM) for improved spectral efficiency," in Intelligent Signal Processing and Communication Systems (ISPACS), 2015 International Symposium on. IEEE, 2015, pp. 552-555.

[34] Y. Chen, L. Wang, Y. Ai, B. Jiao, and L. Hanzo, "Performance analysis of NOMA-SM in vehicle-to-vehicle massive MIMO channels," IEEE Journal on Selected Areas in Communications, vol. 35, no. 12, pp. 2653 2666, 2017.

[35] Z. Wang, J. Cao et al., "NOMA-based spatial modulation," IEEE Access, vol. 5, pp. 3790-3800, 2017

[36] R. F. Siregar, F. W. Murti, and S. Y. Shin, "Combination of spatia modulation and non-orthogonal multiple access using hybrid detection scheme," in Ubiquitous and Future Networks (ICUFN), 2017 Ninth International Conference on. IEEE, 2017, pp. 476-481.

[37] Y. Liu, L. Yang, and L. Hanzo, "Spatial modulation aided sparse codedivision multiple access," IEEE Transactions on Wireless Communications, vol. 17, no. 3, pp. 1474-1487, March 2018.

[38] L.-L. Yang, Multicarrier Communications. Chichester, United Kingdom: John Wiley, 2009.

[39] A. Goldsmith, Wireless Communications. Cambridge University Press, 2005.

[40] W. Abdessamad, Y. Nasser, K. Y. Kabalan, and O. Bazzi, "On the performance evaluation of MIMO-SCMA systems," in Ultra Modern Telecommunications and Control Systems and Workshops (ICUMT), 2016 8th International Congress on. IEEE, 2016, pp. 135-140.

[41] J. Bao, Z. Ma, G. K. Karagiannidis, M. Xiao, and Z. Zhu, "Joint multiuser detection of multidimensional constellations over fading channels," IEEE Transactions on Communications, vol. 65, no. 1, pp. 161$172,2016$.

[42] S.-C. Lim, N. Kim, and H. Park, "Uplink SCMA system with multiple antennas," IEEE Transactions on Vehicular Technology, vol. 66, no. 8 , pp. 6982-6992, 2017.

[43] D. Cai, P. Fan, and P. T. Mathiopoulos, "A tight lower bound for the symbol error performance of the uplink sparse code multiple access," IEEE Wireless Communications Letters, vol. 6, no. 2, pp. 190-193, 2017.

[44] A. Graham, "Kronecker products and matrix calculus: With applications." JOHN WILEY \& SONS, INC., 605 THIRD AVE., NEW YORK, NY 10158, 1982, 130, 1982.

[45] T. J. Richardson and R. L. Urbanke, "The capacity of low-density paritycheck codes under message-passing decoding," IEEE transactions on Information Theory, vol. 47, no. 2, pp. 599-618, 2001

[46] K. Abend and B. D. Fritchman, "Statistical detection for communication channels with intersymbol interference," Proceedings of the IEEE, vol. 58, no. 5, pp. 779-785, 1970.

[47] G. Shafer et al., A mathematical theory of evidence. Princeton university press Princeton, 1976, vol. 1.

[48] W. Ryan and S. Lin, Channel codes: classical and modern. Cambridge University Press, 2009

[49] J. Bao, Z. Ma, M. Xiao, and Z. Zhu, "Error performance of sparse code multiple access networks with joint ML detection," in Vehicular Technology Conference (VTC Spring), 2016 IEEE 83rd. IEEE, 2016, pp. 1-5.

[50] J. Bao, Z. Ma, M. Xiao, Z. Ding, and Z. Zhu, "Performance analysis of uplink scma with receiver diversity and randomly deployed users," IEEE Transactions on Vehicular Technology, vol. 67, no. 3, pp. 2792-2797, 2018.

[51] M. K. Simon and M.-S. Alouini, Digital Communication over Fading Channels, 2nd ed. New York: John Wiley \& Sons, 2005.

[52] M. D. Renzo and H. Haas, "Bit error probability of SM-MIMO over generalized fading channels," IEEE Transactions on Vehicular Technology, vol. 61, no. 3, pp. 1124-1144, March 2012.

[53] R. Zhang, L. L. Yang, and L. Hanzo, "Generalised pre-coding aided spatial modulation," IEEE Transactions on Wireless Communications, vol. 12 , no. 11 , pp. 5434-5443, November 2013.

[54] G. Taricco and E. Biglieri, "Exact pairwise error probability of spacetime codes," IEEE Transactions on Information Theory, vol. 48, no. 2 pp. 510-513, 2002.
[55] T. A. Lamahewa, M. K. Simon, R. A. Kennedy, and T. D. Abhayapala, "Performance analysis of space-time codes in realistic propagation environments: A moment generating function-based approach," Journal of Communications and Networks, vol. 7, no. 4, pp. 450-461, 2005.

[56] P. Pan, L.-L. Yang, and Y. Zhang, "Statistics and error performance of orthogonal frequency-division multiplexing over nakagami-m fading channels," 2011. [Online]. Available: http://users.ecs.soton.ac.uk/lly/ papers/Correlation-Fading-of-OFDM-Nakagami.pdf

[57] J. G. Proakis, Digital Communications, 5th ed. McGraw Hill, 2007.

[58] M. Taherzadeh, H. Nikopour, A. Bayesteh, and H. Baligh, "SCMA codebook design," in Vehicular Technology Conference (VTC Fall), 2014 IEEE 80th. IEEE, 2014, pp. 1-5.

[59] Z. Pan, J. Luo, J. Lei, L. Wen, and C. Tang, "Uplink spatial modulation scma system," IEEE Communications Letters, vol. 23, no. 1, pp. 184 $187,2018$.

[60] E. Telatar, "Capacity of multi-antenna Gaussian channels," European Transactions on Telecommunications, vol. 10, no. 6, pp. 585-595, 1999. 